\title{
AIAA 2001-0159 SENSITIVITY STUDY OF THE WALL INTERFERENCE CORRECTION SYSTEM (WICS) FOR RECTANGULAR TUNNELS
}

Eric L. Walker and Joel L. Everhart NASA Langley Research Center, Hampton, VA 23681

Venkit lyer

Lockheed Martin, Hampton, VA 23681

\section{9th AIAA Aerospace Sciences Meeting and Exhibit January 8-11, 2001/Reno, NV}


AIAA 2001-0159

\title{
SENSITIVITY STUDY OF THE WALL INTERFERENCE CORRECTION SYSTEM (WICS) FOR RECTANGULAR TUNNELS
}

\author{
Eric L. Walker* and Joel L. Everhart ${ }^{\dagger}$ \\ NASA Langley Research Center, Hampton, VA 23681 \\ Venkit Iver ${ }^{\ddagger}$ \\ Lockheed Martin, Hampton, VA 23681
}

\begin{abstract}
An off-line version of the Wall Interference Correction System (WICS) has been implemented for the NASA Langley National Transonic Facility. The correction capability is currently restricted to corrections for solid wall interference in the model pitch plane for Mach numbers less than 0.45 due to a limitation in tunnel calibration data. A study to assess output sensitivity to measurement uncertainty was conducted to determine standard operational procedures and guidelines to ensure data quality during the testing process. Changes to the current facility setup and design recommendations for installing the WICS code into a new facility are reported.

\section{Nomenclature}

$C_{D} \quad$ Nondimensional drag coefficient

$C_{L} \quad$ Nondimensional lift coefficient

$C_{x} \quad$ An output variable

E Error

$M \quad$ Mach number

$N \quad$ Number of ports

$Q \quad$ Dynamic Pressure

$U \quad$ Uncertainty

$U_{e} \quad$ Axial velocity determined by calibration

$U_{\infty} \quad$ Free-stream velocity

$u_{i}, v_{i}, w_{i}$ Velocity perturbation; $X, Y, Z$ components

$X, Y, Z$ Cartesian tunnel axes

$\alpha \quad$ Angle of attark [rad]

* Research Engineer, Research Facilities Branch, Aerodynamics, Aerothermodynamics, and Acoustics Competency. Student Member AIAA.

${ }^{\dagger}$ Research Engineer, Research Facilities Branch, Aerodynamics, Aerothermodynamics, and Acoustics Competency. Senior Member AIAA.

$\ddagger$ Aeronautical Engineer. Senior Member AIAA.

Copyright (C) 2001 by the American Institute of Aeronautics and Astronautics. Inc. No copyright is asserted in the United States under Title 17 , U.S. Code. The U.S. Government has a rovaltyfree license to exercise all rights under the copyright claimed herein for Governmental Purposes. All other rights are reserved by the copyright owner.
\end{abstract}

$\begin{array}{ll}\beta & \text { Angle of sideslip [rad] } \\ \Delta & \text { Correction } \\ \varepsilon & \text { Blockage Factor } \\ \sigma & \text { Population Standard Deviation } \\ \hat{\sigma} & \text { Population Standard Deviation Estimator } \\ \phi & \text { Velocity potential }\end{array}$

\section{Subscripts}

(\%) Percent of value

corr Corrected

$F \quad$ Free air

$i \quad$ incremental correction

$P \quad$ Pressure

$T \quad$ In tunnel

$t \quad$ Total

une Uncorrected

$\infty \quad$ Freestream

\section{Superscripts}

(L) Confidence level

\section{Introduction}

The subject of wall interference has broadened immensely over the past century: Classical correction methods for this effect incorporate a mathematical boundary condition for the wind tunnel walls. Nondimensional parameters have been tabulated and a number of formulas to correct spatially-varying wallinterference for aircraft-like models have been documented in texts such as AGARDograph 109.' More often facilities are testing in regions that exceed the design specifications of the facility. Increased model size, for instance, introduces larger amounts of blockage and lift interference than the classical methods can properly handle. Modern, more capable interference-correction methods, including boundary pressure methods were recently published in AGARDograph $336 .^{2}$ The advantage of the boundary pressure 
methods over classical methods is their ability to respond to the actual conditions of the flow in the tunnel test section; additionally, boundary pressure methods are more complex than classical methods but can be implemented for online post-point/post-scan applications.

Customers are also placing more stringent accuracy requirements on the acquired data. To reduce the uncertainties introduced in tunnel data due to the presence of the walls, thereby enhancing data quality, NASA Langley Research Center has implemented the Wall Interference Correction System (WICS) for solid wall testing in the National Transonic Facility (NTF). ${ }^{3}$ The code was originally developed by Ulbrich et al. ${ }^{49}$ at NASA Ames Research Center for the 12-Foot Pressure Wind Tunnel. The WICS code uses a modified Hackett (Wall Signature) Method to determine wall interference corrections. This method was selected because it is rapid and robust, and it has minimal impact on the facility in terms of instrumentation when compared to other boundary pressure methods. A brief description of the theory behind the WICS method and the implementation at the NTF is presented.

The purpose of this paper is to determine code sensitivities to measurement uncertainty and to establish uncertainty boundaries. Guidelines for implementation and operation are provided based on observations of this study. Although there is error inherent in the theoretical model due to limiting assumptions, this paper does not address the validity of the predicted corrections.

\section{Correction Accuracy Requirements}

Before a sensitivity study can be conducted on the WICS code, it is necessary to understand the accuracy requirements placed on wall interference corrections in general. According to Steinle and Stanewsky, ${ }^{10}$ the requirements for transonic cruise are dominated by a one count $\left(C_{D}=0.0001\right)$ accuracy for drag for a transport-type aircraft. Since the WICS code is not used exclusively on transport-type aircraft in cruise, a more general requirement is needed. Newman and Everhart ${ }^{2}$ report the accuracy requirements listed below in Table 1.

\begin{tabular}{|c|c|c|}
\hline Type of Test & Incremental & Absolute \\
\hline High Lift & $0.2 \% C_{L} \& C_{D}$ & $0.4 \% C_{L} \& C_{D}$ \\
\hline Transonic & $1 / 2$ count $C_{D}$ & 1 count $C_{D}$ \\
\hline
\end{tabular}

Table 1 Generalized Industry Correction Accuracy Requirements

\section{The Wall Interference Correction System (WICS)}

The following presents a brief overview of Ulbrich's extension of the Hackett wall signature method, including theory and the current implementation of
WICS in the NTF. A more detailed description is given by Ulbrich et al..$^{4-9}$ and Iyer et al. ${ }^{3}$ The method applies a measured pressure boundary condition which is the tared difference between the model installed condition and the empty tunnel. The test article is comprised of singularities: a source-sink combination is used to represent the fuselage; sources are used to represent the viscous separation in the wake; and line doublets, typically placed on a lifting surface quarter-chord, are used to simulate the effect of lift. Additionally, a powered simulation can be represented by proper placement of a sink. Once the strengths of the line doublets and propulsion simulation sinks are known, the signature of these singularities is subtracted from the tared wall signature. The remaining signature is used to determine the strengths of the solid and wake blockage singularities. Hackett et al. 11,12 originally used a "local" least squares fitting procedure of the wall pressure data, which was labor intensive because each pressure measurement used in the fit of the wall signature had to be individually selected for each data point. In an effort to improve the process, Ulbrich $^{9}$ introduced a "global" least squares fitting procedure that uses all available pressure ports. The use of panel codes to precompute normalized solutions for use in matching the signature was also introduced. Ulbrich further modified the Hackett method by using balance measured forces and moments to calculate the strength of the doublet singularities. The method uses tared data to assess the wall induced effects on the test article. The tared data are obtained by subtracting empty tunnel wall pressure port calibrations from the model-installed pressure data to remove first order effects of the empty tunnel boundary layer and buoyancy. This taring of the measured data assumes that the additional second order change in the boundary layer displacement thickness due to the model in the tunnel is negligible. Normalized perturbation velocities are calculated from the subsonic potential equation using the method of images, for each type of singularity in discrete locations in the tunnel. By superimposing these model singularities with their corresponding calculated strengths, interference velocities are computed.

The blockage interference factor $\varepsilon$ is defined by

$$
\varepsilon=\frac{u_{i}}{U_{f}} \approx \frac{u_{T}-u_{F}}{U_{e}}
$$

where $u_{i} / U_{e}=\partial \varphi_{I} / \partial x$ and $\varphi_{I} \approx \varphi_{T}-\varphi_{F}$. Incidence and sideslip corrections are defined by

$$
\begin{aligned}
\alpha_{i} & =\frac{U_{e}}{U_{\infty}} \frac{w_{i}}{U_{e}} \approx \frac{U_{e}}{U_{\infty}} \frac{w_{T}-w_{F}}{U_{e}} \\
\beta_{i} & =\frac{U_{e}}{U_{\infty}} \frac{v_{i}}{U_{e}} \approx \frac{U_{e}}{U_{\infty}} \frac{v_{T}-v_{F}}{U_{e}}
\end{aligned}
$$

where $U_{\infty} / U_{e}$ is defined by $1+\varepsilon$. It is important to note that the integrated effect of the empty tun- 
nel boundary layer growth and buoyancy due to improper wall divergence are already included in $U_{e}$. The "empty-tunnel" calibration provides the perturbation velocity denoted by $u_{\delta}$, which can be used to correct the measured model-in-tunnel perturbation velocity. $u_{T}^{\prime}$, by

$$
\frac{u_{T}}{U_{e}}=\frac{u_{T}^{\prime}-u_{\delta}}{U_{c}}
$$

The ratio $u_{T} / U_{e}$ is used to determine the singularity strengths.

\section{General Implementation Requirements}

To implement the WICS code, ${ }^{3}$ several tasks must first be completed. The first task is to ensure the facility has an adequate number of static pressure orifices on the wall of the test section. (The meaning of adequate will be discussed later in this paper.) Next, a perturbation velocity database (PVD) must be generated using a panel method or the method of images. This PVD is used in the matching of the tared wall signature to determine singularity strengths, and to generate the field of interference velocities so that corrections can be computed.

Once the orifices have been installed, an "empty tunnel" calibration must be performed. This calibration is a function of independent test parameters (e.g., total pressure and Mach number) and support system attitude (e.g., pitch and sideslip angles). For semispan models, the empty tunnel calibration is the tunnel geometry minus the model. For fullspan models the model support system is included, and the sting or support system kinematics must also be considered. Semispan and fullspan model installation details are provided subsequently.

\section{Code Output Results}

Figures 1 - 3 present a sample of the WICS code output from the NTF for a semispan model. Figure 1 gives a row-wise comparison of the tared signature with the WICS-fit wall signature for a single test point at $M=0.2, \alpha_{\text {unc }}=24^{\circ}$ for a semispan model. The symbols represent measured, tared wall perturbation velocities and the solid line represents the global least squares prediction of the code. (One measure of validity is how well these two match.) Mean primary corrections are displayed in Figure 2. These corrections are shown for an entire pitch polar (or group of data points). Blockage corrections ( $\varepsilon$ ) are averaged interference velocities along the fuselage centerline, and upwash (incidence) corrections $\left(\alpha_{i}\right)$ are averaged along the wing three-quarter-chord. Corrections to Mach number $(\Delta M)$ and dynamic pressure $(\Delta Q)$ are derived from the blockage factor. Coefficient corrections for the entire polar are plotted in Figure 3. Note for the test point of $\alpha=24^{\circ}$ in Figure 1 that significant corrections of $\Delta M=0.0025$ and $\alpha_{i}=1.4$ are determined as shown in Figure 2. Correspondingly large corrections for the coefficients are also observed (Figure 3). Since the WICS code calculates the interference corrections for specified reference planes in a volume surrounding the model, contours of the correction variation can be plotted.

\section{Description of the NASA LaRC National Transonic Facility}

The NTF ${ }^{13}$ is a fan-driven, closed-circuit. continuous-flow, pressurized wind tumnel, which is rapable of testing at cryogenic conditions. The test gas is dry air for elevated temperature operation and nitrogen for reduced temperature operation. The settling chamber contains four anti-turbulence screens. A 15:1 contraction ratio entrance cone leads into an 8.2 feet square cross sectional test section with six inch triangular corner fillets which extends 25 feet in length then opens into a high speed diffuser. The operational envelope of the NTF encompasses a large range of test conditions. The facility can sustain a continuous airspeed from 0.1 to 1.2 in Mach number. Total pressure capabilities of the facility range from 15 to $130 \mathrm{psi}$. The tunnel can operate at temperatures ranging from $150^{\circ} \mathrm{F}$ down to $-320^{\circ} \mathrm{F}$. These large ranges of conditions allow Reynolds number testing from 3 to 120 million per foot. NTF has the capability to independently vary Mach number, Reynolds number, and dynamic pressure.

Both fullspan and semispan model mounting systems are available in the NTF. Fullspan models are supported by an aft mounted sting. This sting is attached to a vertically oriented arc sector used to change model pitch attitude over a range from $-11^{\circ}$ to $19^{\circ}$. The roll mechanism has a range of $-90^{\circ}$ to $180^{\circ}$. Sideslip angles are achieved by using combined roll and pitch angles. A sidewall mounting system is used for semispan models. The angle of attack range is $\pm 180^{\circ}$. The center of rotation for semispan and fullspan models is tunnel station 13 feet.

Currently, the NTF has 459 operational wall pressure orifices of which the WICS code uses 360 . Looking downstream, Figure 4 shows the cross sectional diagram of the rows of pressure ports; it also serves as a map between the NTF row numbering scheme and that used for WICS. Figure 5 unwraps the tunnel walls to show the current wall orifice layout. The filled port symbols are an example subset of port selections used for WICS. ${ }^{3}$

\section{Approach and Rationale}

The original approach to define the WICS code sensitivities to measurement uncertainty was to use actual test data, perturb it using a known and controlled random error and bias, and evaluate the output. Following this path is equivalent to declaring an existing data set to have zero uncertainty. This method was rejected because it gave no easy method for assessing 
the magnitude of instrumentation error inherent in the current test data. Assessment of this error standard deviation is part of the ongoing Data Quality Assurance Program at LaRC conducted by Hemsch et al. ${ }^{14}$

An alternate method was devised to obtain an idealized set of data from actual test data. The code, as prart of the solution process, generates a global least squares fit of the wall signature. Additional coding was added to WICS to enable the discretization of this wall signature fit. This result is reinput and a new fit of the wall pressure data is computed, thereby creating a data set with no random error. This new wall signature and the corresponding test data are used as a baseline for the sensitivity study. In terms of the WICS calculation, force, moment, and reference condition data are point measurements. Therefore, only the wall pressure data were deemed necessary to be fitted (smoothed) to remove noise. The idealized data set assumes that there are no second order boundary layer or velocity perturbation effects, such as model induced nonuniformities in the tumnel wall boundary layer. Four test articles were used in this study; however, all figures shown refer to a low aspect ratio, high lift, semispan model. A more detailed description of this analysis is given by Walker ${ }^{15}$ including results from the other three test articles.

The code has four major categories of error sources: the wall pressure signature, force and moment data, tunnel reference condition data, and the WICS mathematical model. Given this information, the total uncertainty in the code output can be written as:

$$
U_{t}=f\left(U_{W P S}, U_{F / M}, U_{R E F}, U_{W I C S M} \text { odel }\right) .
$$

Each of the four categories in Equation 5 can be expanded. First, the wall signature is composed of precision and bias errors due to the wall pressure instrumentation, thus yielding:

$$
U_{W P S}=f\left(P_{W P S}, B_{W P S}\right) .
$$

Second, force and moment data consist of calculated lift, drag, and side force; roll, pitch, and yaw moments; and measured angle of attack.

$$
U_{F / M}=f\left(U_{L F}, U_{D F}, U_{S F}, U_{P M}, U_{Y M}, U_{R M}\right) .
$$

Third, the tunnel reference conditions, which in the NTF are determined from the Mach number reference system, will be examined.

$$
U_{R E F}=U_{M A C H}
$$

Last, the WICS model incorporates the singularity representation, setup information, and the global least squares fitting process. The inherent error in the WICS model is beyond the scope of this paper and the corresponding uncertainty term is assumed to be zero or negligible for this study. A discussion of the remaining uncertainties is the subject of the remainder of this paper.

\section{Wall Pressure Measurements}

The NTF wall signature used for WICS computation currently consists of a total of 360 wall pressure orifices distributed in rows on the four walls. In Equation 6 it was stated that the uncertainty in the wall pressure signature could be broken into a precision error and a bias error. Precision error is defined as the random normal variation of a measured parameter about some expected value, whereas bias error is defined as the difference between some measured expected value and the "true value" of a parameter. The following sections will evaluate the effects of these errors. As will be discussed, the magnitude of precision error is used to establish proper radial placement of rows, while the magnitude of bias error establishes the orifice distribution within the rows.

\section{Random Noise (Precision Error)}

From elementary statistics, a confidence interval is defined as the distance from the measured mean within which the true mean should lie given a specified confidence level or

$$
C I^{(L)}=\bar{x} \pm \frac{Z^{(L)} \hat{\sigma}}{\sqrt{N}}
$$

where $\bar{x}$ is the measured mean, $\hat{\sigma}$ the measured standard deviation of $N$ samples or measurements and $Z$ the standard normal statistic at a confidence or significance level of $L$. Make note that when the number of samples is less than $30, Z$ is no longer standard normal but follows the student $t$ distribution. ${ }^{16}$ For simplicity, the standard normal distribution is assumed to hold for all values of $N$. A confidence interval can also be thought of as the measured mean with some error band at a given significance level, written as

$$
\bar{x} \pm E^{(L)}
$$

If $\sigma_{P}$ is defined as the standard deviation of the wall pressure measurements, an expression for the error band of the wall pressures may be written

$$
E_{P}^{(L)}=\frac{Z^{(L)} \sigma_{P}}{\sqrt{N}}
$$

Choosing a $99.72 \%$ significance level or a standard normal statistic of approximately 3 for data quality assurance purposes, the expected error band can be predicted for a range of $\sigma_{P}$ and $N$. Note that choosing a $99.72 \%$ significance level equates to one erroneous measurement out of approximately 350 ; whereas a $95 \%$ significance level is equivalent to one out of 20 .

An estimate of the value of $\sigma_{P}$ for the NTF is 0.002 psi. This value was inferred from output of the tunnel on-line data system for low Mach numbers (0.1 to 0.3 ).

\section{Random Seeding}

If the confidence interval is tight around the expected value calculated from measured data, the true 
mean is confidently and accurately predicted. For this case, at a fixed $\hat{\sigma}$, the predicted mean should be insensitive to random seeding of the random number generator used to introduce a prescribed measurement error. Increasing $\hat{\sigma}$ in Equation 9 with fixed $Z^{(L)}$ and $N$, will, at some value of $\hat{\sigma}$, cause the conficlence interval to become so wide that the mean cannot be predicted with accuracy. At this point the predicted mean will be increasingly sensitive to the effects of random seeding.

Based on results presented by Walker, ${ }^{15}$ the wall signature is insensitive to error due to random seeding for $\sigma_{P}<0.05 \mathrm{psi}$; beyond this, the wall signature cannot be accurately predicted. The global least squares fitting routine is able to adequately recover the underlying character of the pressure signature, thereby yielding a robust prediction technique. For values of $\sigma_{P} \geq 0.05 \mathrm{psi}$, the seed yielding the largest deviated case was conservatively selected to minimize an introduction of additional error in later analysis.

Variation of $\sigma_{P}$

Using the typical value of $\sigma_{P}$ as a guideline, six values, ranging from 0.001 to 0.1 psi, encompassing three orders of magnitude were used to define the code sensitivity to variation of $\sigma_{P}$. The wall signature is not very sensitive to random perturbation as discussed in the previous section. The insensitivity of wall pressure to random perturbation translates, as would be expected. into output insensitivity as evidenced in the incremental blockage results presented in Figure 6. This figure is representative of the sensitivities of each output variable examined over the data run for various values of $\sigma_{P}$. The maximum and average values for the data run are shown. Figure 6 a shows the scatter of incremental blockage error for the entire data run, and Figure $6 \mathrm{~b}$ shows a linear-logarithmic plot of the maximum and mean values for each value of $\sigma_{p}$. The solid and dashed lines represent a linear fit of the maximum and average values for each value of $\sigma_{P}$, respectively. This figure is representative of all the output and on this logarithmic plot it shows a strong positive linear correlation between the absolute and average error in the output variables and $\sigma_{P}$. It is also evident that the distance between the fit of the maximum and mean errors becomes larger as $\sigma_{P}$ increases, demonstrating that the scatter in the error becomes large with relatively large $\sigma_{P}$.

\section{Variation of $N$ (Loss of Pressure Data)}

A precision-sensitivity model that will enable the prediction of output error due to random noise in the wall signature can be developed as follows. It is understood that

$$
E_{C_{x}^{\prime}}^{(L)}=f\left(E_{P}^{(L)}\right)
$$

where $C_{x}$ is any output variable. If $f$ is assumed linear due to the strong linear correlation as seen in Figure 6 ,
Equation 12 may be written as

$$
E_{C_{x}}^{(L)}=a E_{P}^{(L)}+b
$$

Since this portion of the study is only concerned with $E_{p}^{(L)}$, it follows that $b=0$, which implies that when there is no error in the wall pressure system there is no error in the output variables. Thus, this model will only predict output error due to random noise in the wall signature. Using Equation 11. Equation 13 may be ro-expressed as

$$
E_{C}^{(L)}=a \frac{Z^{(L)} \sigma_{\Gamma}}{\sqrt{N}}
$$

Solving Equation 14 for $N$ yields

$$
N=\left[\frac{a Z^{(L)} \sigma_{P}}{E_{C_{s}}^{(L)}}\right]^{2}
$$

Equation 14 allows $E_{C_{x}}^{(L)}$ to be predicted for variation in $N$, and Equation 15 allows for the prediction of the number of required pressure ports for a given $\sigma_{P}, Z^{(L)}$, and $E_{C_{x}}^{(L)}$

By regressing the maximum values of error for each of the output variables, a conservative estimate of the value of $a$ for Equation 13 may be determined for each output variable. Table 2 tabulates these values. Using Table 2 and Equation 15, prediction plots at the $99.5 \%$ confidence level were made corresponding to several of the output variables. Figures 7 and 8 show the predicted error for the primary corrections $\varepsilon$ and $\alpha_{i}$, respectively. In these figures, the predicted number of ports required is plotted against $\sigma_{P}$ for lines of constant error in the output variables. This allows the determination of a required number of ports for a given $\sigma_{P}$ and a defined maximum error level. Make note that the units of each error level correspond to the units of the output variable. As an example, for the NTF typical $\sigma_{P}=0.002 \mathrm{psi}$, the number of ports required to ensure incidence correction error prediction below $0.005^{\circ}$ is 240 . The corresponding number to ensure that the blockage correction is missed by no more than $0.01 \%$ is 120 . Accuracy of the angle of attack is obviously the dominant criteria for selecting the number of ports based on error in primary corrections, while blockage requirements can be achieved with a relatively small number. In accordance with the generalized requirements placed on testing in Table 1, Figure 9 gives the predicted error in terms of $\Delta C_{D_{t}}$, and Figures 10 and 11 give the predicted error in terms of $\Delta C_{L(\%)}$ and $\Delta C_{D_{\mathrm{t}}(\%)}$, respectively.

\section{Row Placement}

The eight semispan and ten fullspan wall pressure orifice configurations defined in Tables 3 and 4 , respectfully, were used to evaluate output sensitivity due 


\begin{tabular}{|c|c|}
\hline Variable & $a$ \\
\hline \hline$\varepsilon$ & 0.1887 \\
\hline$\Delta M$ & 0.0382 \\
\hline$\Delta Q$ (psf) & 129.51 \\
\hline$\alpha_{i}$ & 2.632 \\
\hline$\Delta C_{L}$ & 0.0118 \\
\hline$\Delta C_{L(\%)}$ & 1.652 \\
\hline$\Delta C_{M}$ & 0.0167 \\
\hline$\Delta C_{D}$ & 0.0349 \\
\hline$\Delta C_{D_{b}}$ & 0.0317 \\
\hline$\Delta C_{D_{t}}$ & 0.0382 \\
\hline$\Delta C_{D(\%)}$ & 14.065 \\
\hline$\Delta C_{D_{b}(\%)}$ & 17.461 \\
\hline$\Delta C_{D_{t}(\%)}$ & 14.868 \\
\hline
\end{tabular}

Table 2 Regressed Values for the Coefficient $a$ in Equation 15 for Prediction of Error in Output Variables due to Variation in $\sigma_{P}$ and $N$

\begin{tabular}{|c|l|l|l|}
\hline Case & Name & WICS Rows & N \\
\hline 1 & Full_Sig & $1-5,7-12$ & 260 \\
\hline 2 & Alternating & $1,3,5,7,9,11,12$ & 161 \\
\hline 3 & Mirror_CLine & $5,7,12$ & 67 \\
\hline 4 & Tunnel_CLine & $3,9,12$ & 64 \\
\hline 5 & Far_Field & $1,11,12$ & 62 \\
\hline 6 & Min_Top & 7 & 26 \\
\hline 7 & Min_Bott & 5 & 25 \\
\hline 8 & Min_Side & 12 & 16 \\
\hline
\end{tabular}

Table 3 Semispan Row Hookup Configurations

\begin{tabular}{|c|c|c|c|}
\hline Case & Name & WICS Rows & $\mathrm{N}$ \\
\hline 1 & Full_Sig & $1-12$ & 290 \\
\hline 2 & Alternating & $1,3,5-7,9,11,12$ & 191 \\
\hline 3 & FourCorner_Side & $1,5-7,11,12$ & 143 \\
\hline 4 & FourCorner & $1,5,7,11$ & 97 \\
\hline 5 & Tunnel_CLine & $3,6,9,12$ & 94 \\
\hline 6 & $14 \times 22 \_$Sim & $6,9,12$ & 72 \\
\hline 7 & Min_Far_Side & 6 & 30 \\
\hline 8 & Min_Top & 9 & 26 \\
\hline 9 & Min_Bott & 3 & 22 \\
\hline 10 & Min_Near_Side & 12 & 16 \\
\hline
\end{tabular}

Table 4 Fullspan Row Hookup Configurations

to row placement configurations and to verify predictions in Figures $7-11$ at various values of $\sigma_{P}$. These configurations include the full measurement, near field (radially), far field (radially), and single row resolutions of the wall signature. In addition, approximations to other facility configurations were evaluated. Walker ${ }^{15}$ showed that the error predicted in Figures 7 - 11 is a fairly conservative estimate with notable exceptions due to orifice placement.

In the random perturbation of the wall signature, the doublet strengths which define the lift influence are not affected. Therefore, all randomness is transferred to the blockage component of the pressure signature. and the blockage factor will be more sensitive to this type of experimental noise. The error in blockage increases with the decrease in the number of active ports. which corresponds to a less accurate fit of the singularity strengths. Error in the blockage factor was found to be approximately the same order of magnitude for numbers of ports greater than or approximately equal to 150 . Row configurations with less than 150 ports are therefore less desirable. Since the upwash correction incorporates both blockage and lift interference, the overall trends in the upwash correction factor will differ slightly from those of the blockage. As with the blockage correction, the magnitude of error in the upwash correction tends to be approximately the same for cases in which the number of ports is greater than approximately 150 . This again suggests that row configurations with less than 150 ports are inadequate. unless special care is taken in the placement of rows and orifices. It is also important to note that the measured pressure signature from rows of orifices in the spanwise far-field of the model yield a higher level of error than those rows positioned near the model. This is primarily due to the radial distance of the ports from the singularity representation of the model. Given these trends, it is recommended that the majority of the orifices be located in the vicinity of the model. It is noteworthy to point out that a higher average error exists for measurements from rows of pressure orifices placed directly below the model as opposed to directly above the model. This suggests a slight favoring of the ceiling orifices, and in terms of maintenance and orifice integrity, ceiling pressure ports are favorable. Rows of pressure orifices in the spanwise far-field and small total numbers of orifices yield less accurate results due to the inability of these configurations and numbers of ports to adequately resolve the wall signature. This results in the inability of the method to accurately differentiate between the lift and blockage interference in these instances. While some of these rows are less important for pitch-plane corrections, the sidewall rows will become essential when full three-dimensional corrections are implemented.

\section{Bias}

Bias error was introduced into the wall pressure sy:tem by adding a constant amount to each wall pressure measurement. The instrumentation used to record wall pressure data has a quoted accuracy of \pm 0.0025 psi; additionally, bias of this magnitude was observed during the acquisition of data used for this study. This range is similar to the precision error case; therefore, the same three orders of magnitude study was conducted to determine sensitivities of the code to bias error. Two separate studies related to bias error were conducted: a global biasing (i.e., the entire wall signa- 
ture) and selective biasing of chosen pressure modules.

\section{Global Biasing}

Biasing of the wall signature on a global scale has the same effect on blockage as changing the Reynolds number; when bias is increased the measured blockage displays an apparent decrease. When Reynolds number increases, the tunnel wall boundary layers thin and blockage decreases. This suggests that bias error in the wall pressures can manifest as a pseudo-Reynolds number effect. Figure 12 shows the biased wall signature for two values of $B_{P}$ as compared to the baseline case. This figure shows the biased wall signatures shifted from the baseline case and gives the corresponding fit of each case. As demonstrated by Walker, ${ }^{15}$ the blockage is affected significantly by bias error; whereas the upwash correction contains only a slight variation. Figure 13 shows the sensitivity of the primary corrections to an increase in $B_{P}$. In contrast to the random noise study, a minimum error now exists because the measured values have been shifted away from the true solution. Very little difference in the blockige is seen in Figure 13a, but the upwash correction in Figure 13b contains a significant difference between the maximum and minimum values of error for each level of bias. This difference in the upwash is due to the fact that the upwash correction contains the superposition of both lift and blockage interference, and any differences seen in the blockage factor are magnified in the upwash correction. Figure 14 shows the error in terms of the force coefficients in percentage of the corresponding uncorrected coefficient, and Figure 15 shows the change in the coefficients. These figures can be used to determine the level of bias allowed according to the error levels in the general requirements for testing (see Table 1). Error in both lift and drag corrections has a strong linear correlation with $B_{P}$.

The effect of the bias is clearly seen in the following example. Several repeat data runs were done during the test of a low-aspect ratio, high-lift, semispan model. Normally, before each set of data runs the wall pressure system was fully calibrated while the tunnel was offline. Calibrations performed during a run are zero calibrations. A full calibration entails fitting a fourth order curve through five data points dispersed over the testing range. During a zero calibration the curve is assumed to have shifted and only the constant or zero term is adjusted for offset, the other coefficients remaining constant. For one particular run the reference pressure drifted when the tunnel came online and only a zero-calibration of wall pressure system was conducted prior to taking data (the full calibration of the wall pressures was performed while the tunnel was offline). For this case it resulted in a biasing of the wall pressure data. During the same data run another zero calibration was performed which unbiased the data. Corrections were computed for these data. Figure 16a shows the blockage results for the three repeat data runs. The step change in the data at $\alpha \approx 14^{\circ}$ plotted with deltas are the results where the zero calibration was performed during the run. After calibration the offset blockage results collapse and follow the trends of the two other repeat runs. Figure 16b shows three repeat runs where a full calibration was performed offline with no zero calibration when the tunnel came online and no problems were observed. Although this biasing phenomena can be duplicated with artificial error, further investigation should be done into the calibration process of wall pressures. The zero calibration taken before acquiring data was probably done before the static reference pressure reached equilibrium and thereby biased the data. When the second zero calibration was performed the static reference pressure had probably equilibrated and the bias was then adjusted out.

Another significant outcome of the global biasing of the wall pressure data is that of port placement for testing. By subtracting the fit of the wall pressures from the biased wall pressures, the ports which exert the most influence on the wall fit can easily be sen. This effect can also be seen in Figure 12 by comparing the biased data with its corresponding WICS fit. The areas where the fit has relatively large deviation from the data are upstream of the model. The data from the ports in these areas of large error had an insignificant effect on the fit generated by the code. Therefore an optimal port configuration for using the WICS code would be to have a vast majority of the pressure ports in the vicinity of the model and approximately one fuselage length downstream where the wake is influential. The primary reason for this is the distance from each port to singularity representation dictates how much influence that port has on the final output. This also suggests that tunnel aspect ratio will have some effect on the fitting of the wall signature since the distance from the model representation singularities to the pressure ports may be larger or smaller.

\section{Selective Biasing}

Selective biasing was performed by adding a pressure increment to all the data from select pressure sensing modules. It can be inferred from results presented by Walker, ${ }^{15}$ that error due to biasing becomes smaller with fewer ports being biased so that the bias is in effect averaged out over the other ports. This is seen predominately in the error of the blockige factor, with one notable exception that the upwash correction seems to be more sensitive to a biasing of the floor pressures than the ceiling.

\section{Force and Moment Measurements}

Of all the forces and moments measured on the model, lift is the primary input to the WICS code. As stated previously, the lift force is used to calcu- 
late the strengths of the line doublets. This means that error in the lift force will cause more error in the upwash correction than blockage. Figure $17 \mathrm{ev}-$ idences this statement by showing resultant error in the primary corrections due to introducing error in lift according to the generalized industry requirements in Table 1. Here, the blockage and upwash correction errors, $\delta \varepsilon$ and $\delta \alpha_{i}$ are plotted against $\alpha_{u n c}$ for error in measured lift of $0.2 \%$ and $0.4 \%$. These lift errors are insignificant for blockage; however, they can achieve as murh as $50 \%$ of the allowable error for angle of attack
$U_{F / M}$ and $U_{R E F}$ terms become negligible and drop from Equation 5. This allows the total uncertainty to be approximated by Equation 6. Then

$$
U_{t} \approx U_{W P S}=f\left(P_{W P S}, B_{W P S}\right)
$$

The combination of errors is done according to the root sum square method (RSS). With the use of this method, any error less than half the size of a larger error is negligible. According to results presented in the section on wall nressure measurements. the amount

${ }^{13}$ Fuller, D., Gloss, B., and Nystrom, D., "Guide for Users of the National Transonic Facility," NASA TM 83124, July 1981.

${ }^{14}$ Hemsch, M., Grubb, J., Krieger, W., and Cler, D., "Langley Wind Tunnel Data Quality Assurance-Check Standard Results," AIAA Paper 2000-2201, 2000.

${ }^{15}$ Walker, E., Sensitivity of the Wall Interference Correction System to Measurement Uncertainty, Master's thesis, The George Washington University, 2000.

${ }^{16}$ Coleman, H. and Steele, W., Experimentation and Uncertainty Analysis for Engineers, Wiley-Interscience, 1989. 


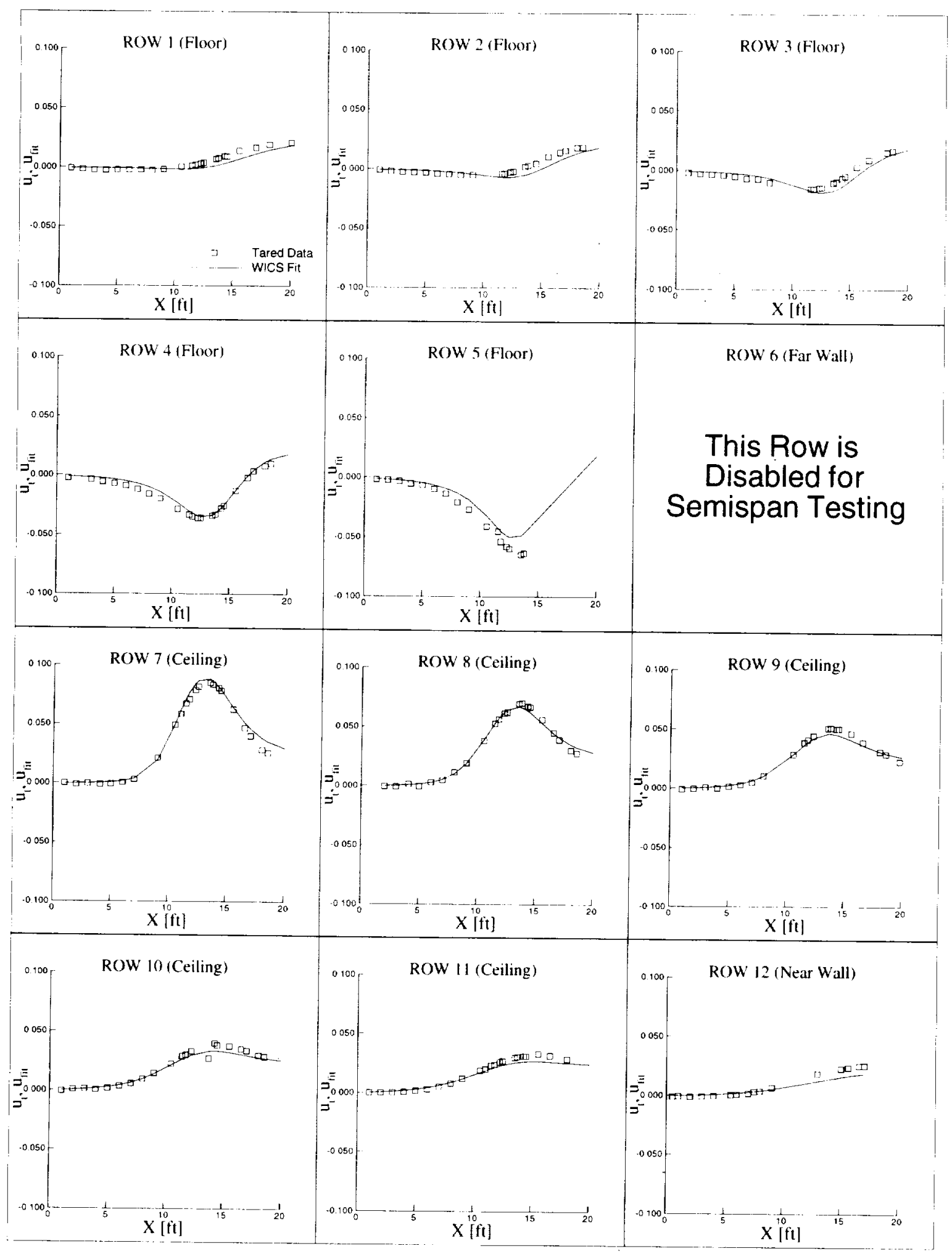

Fig. 1 Wall Signature of a Model at $\alpha_{u n c}=24^{\circ}$

11 OF 27 

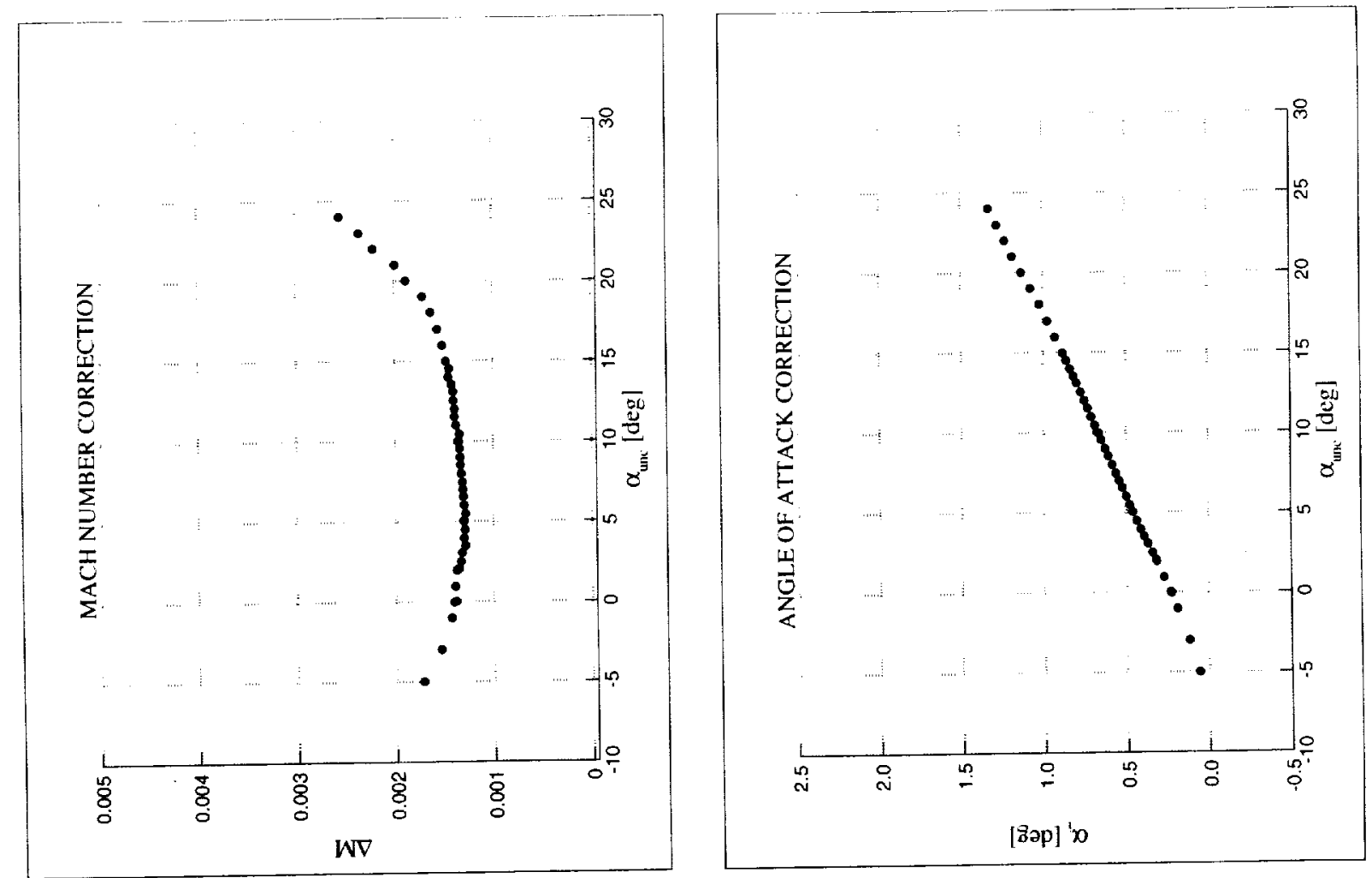

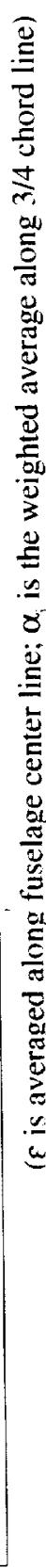

Fig. 2 Mean Primary Corrections

12 of 27 

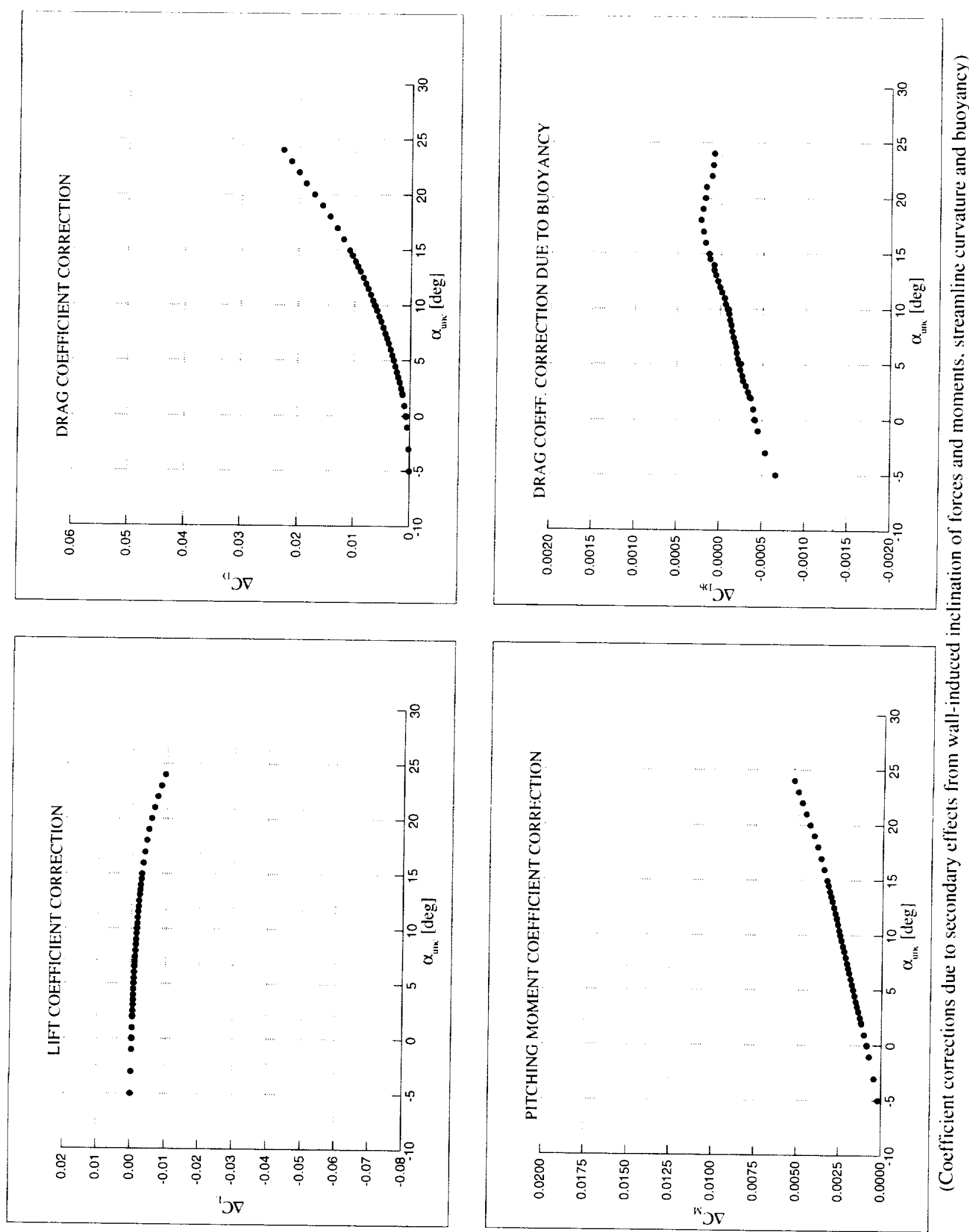

Fig. 3 Coefficient Corrections 


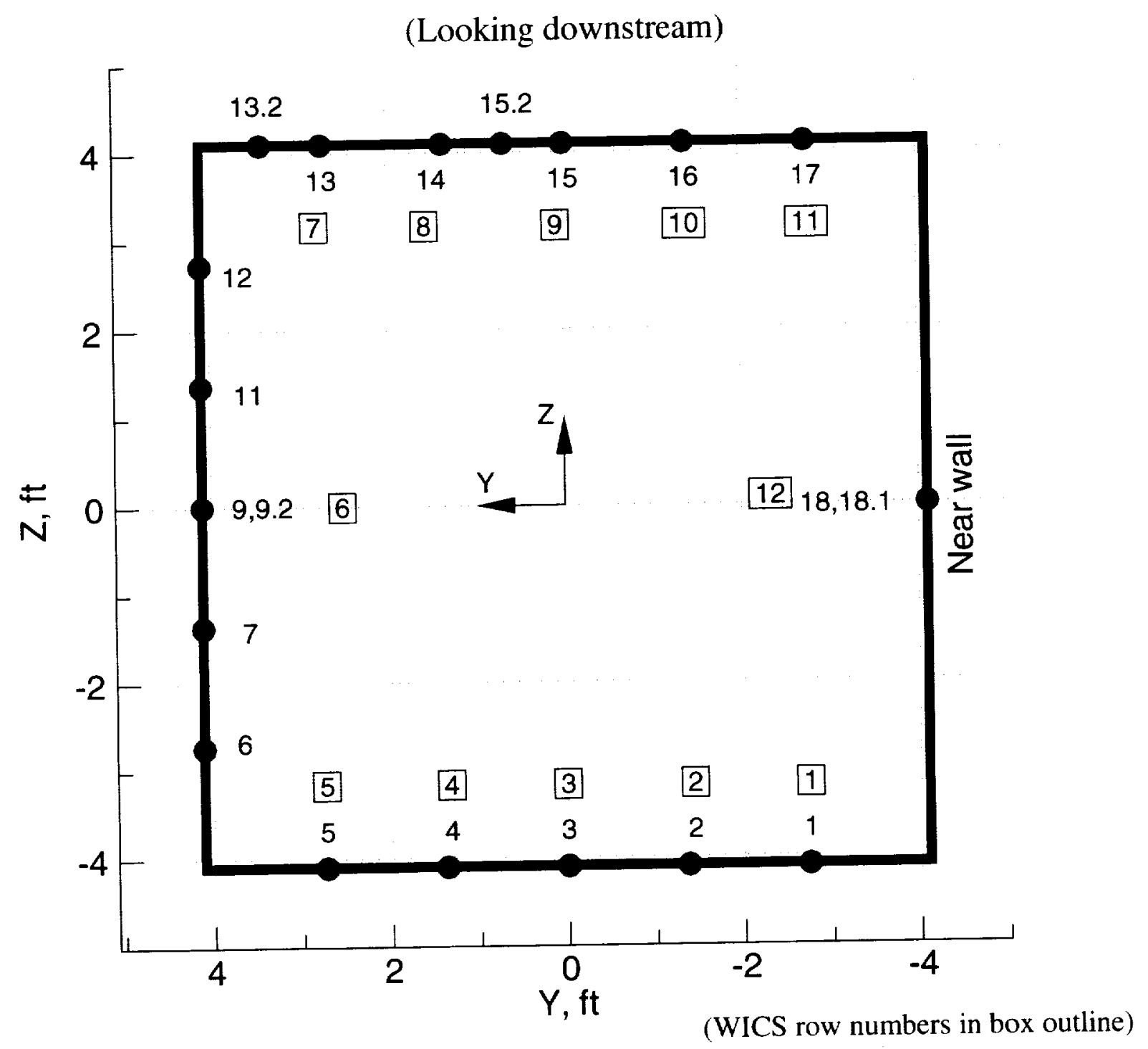

Fig. 4 Cross-Sectional Pressure Measurement Row Setup of the NTF 


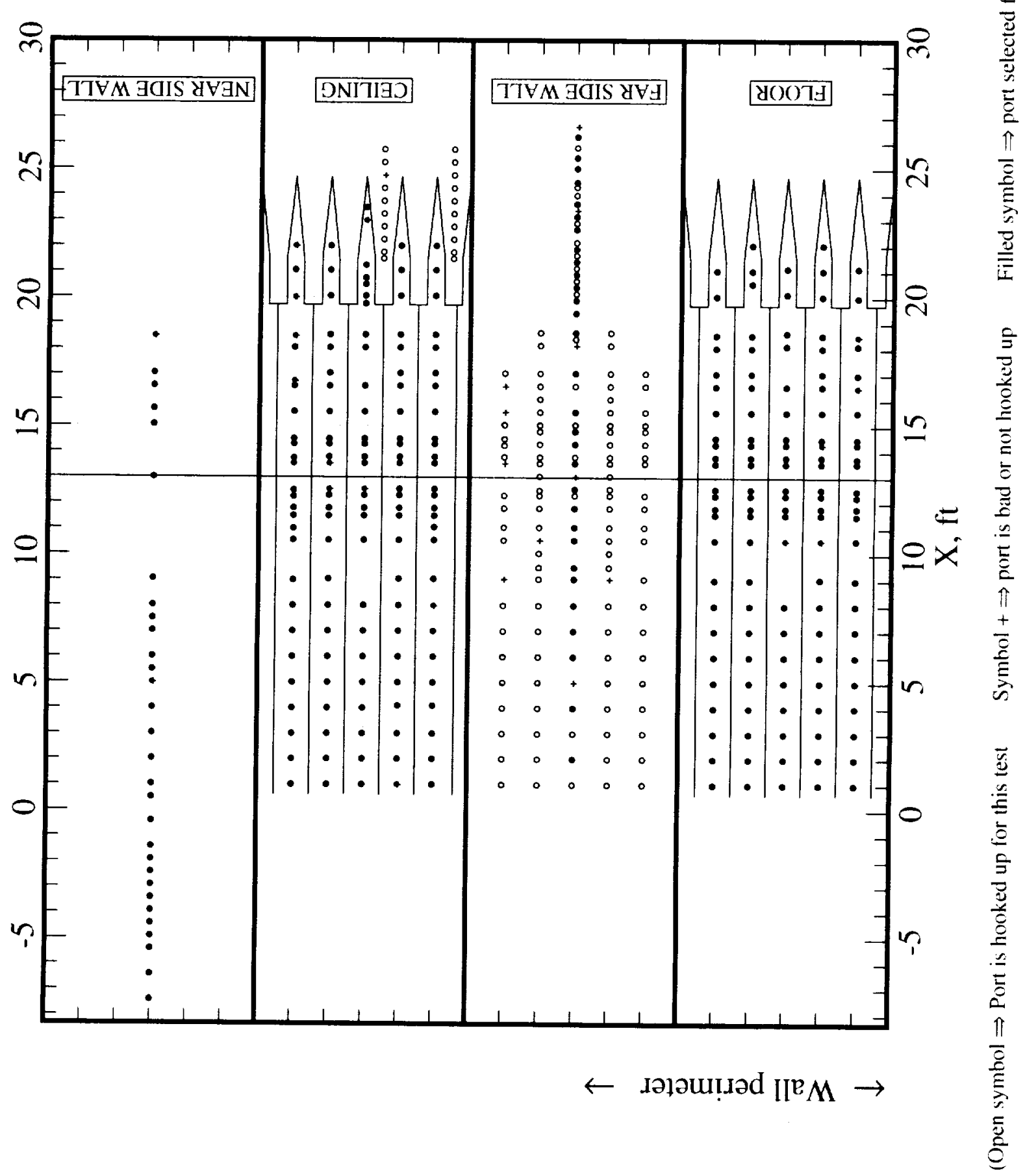

Fig. 5 Wall Orifice Layout for NTF 


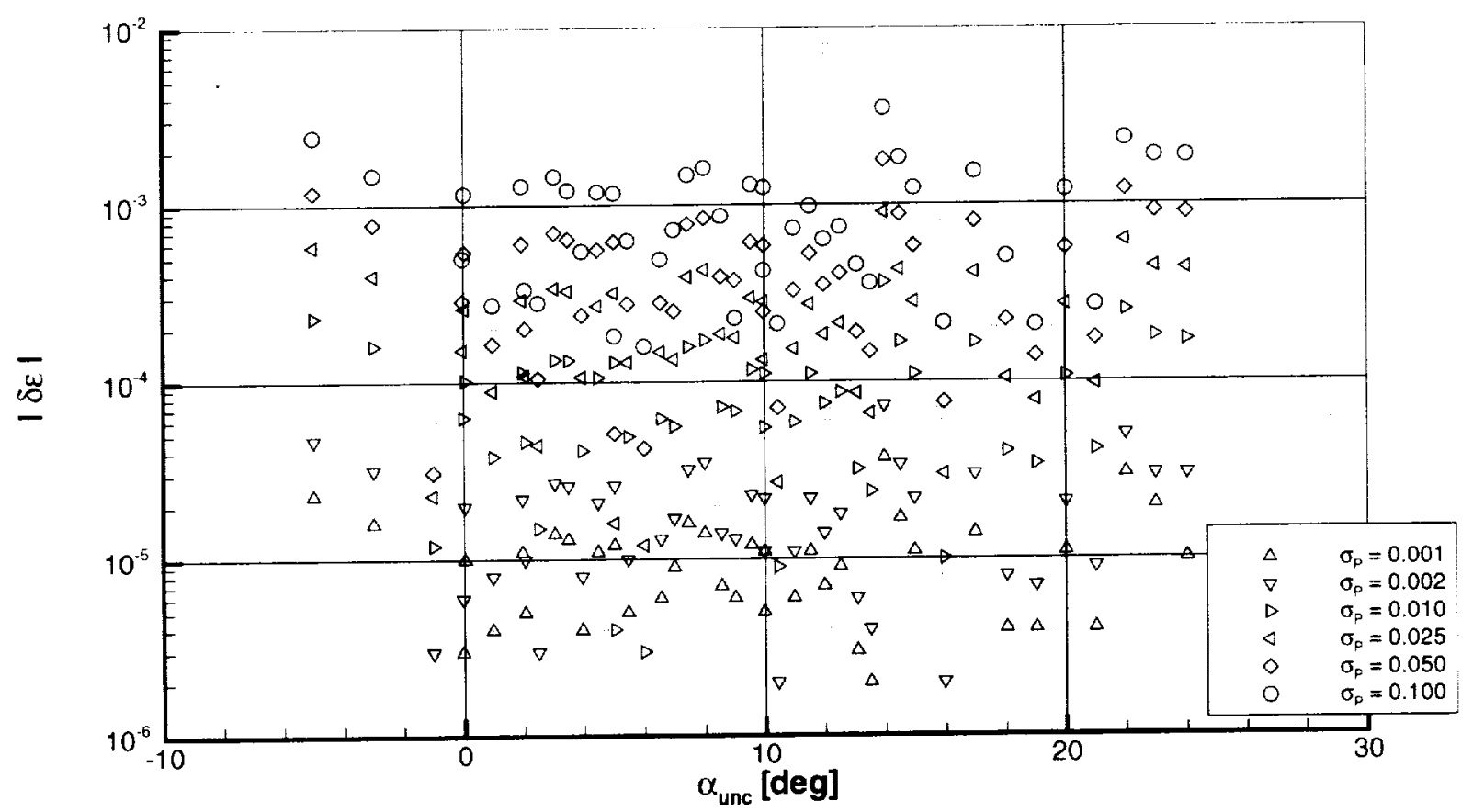

(a) Results for entire data run

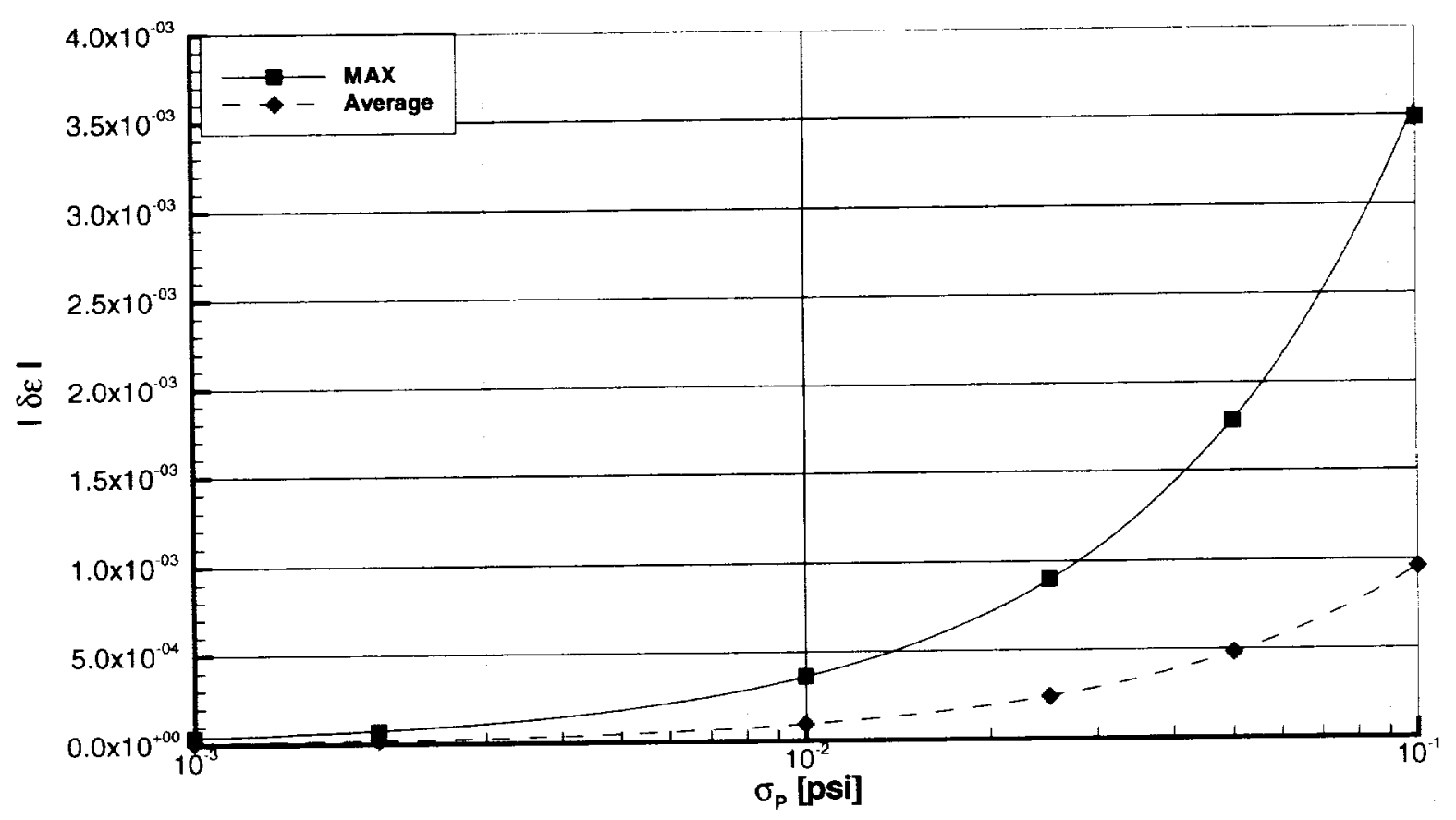

(b) Maximum \& Average Values and Trends

Fig. 6 Error in $\varepsilon$ due to $\sigma_{P}$ Variation 


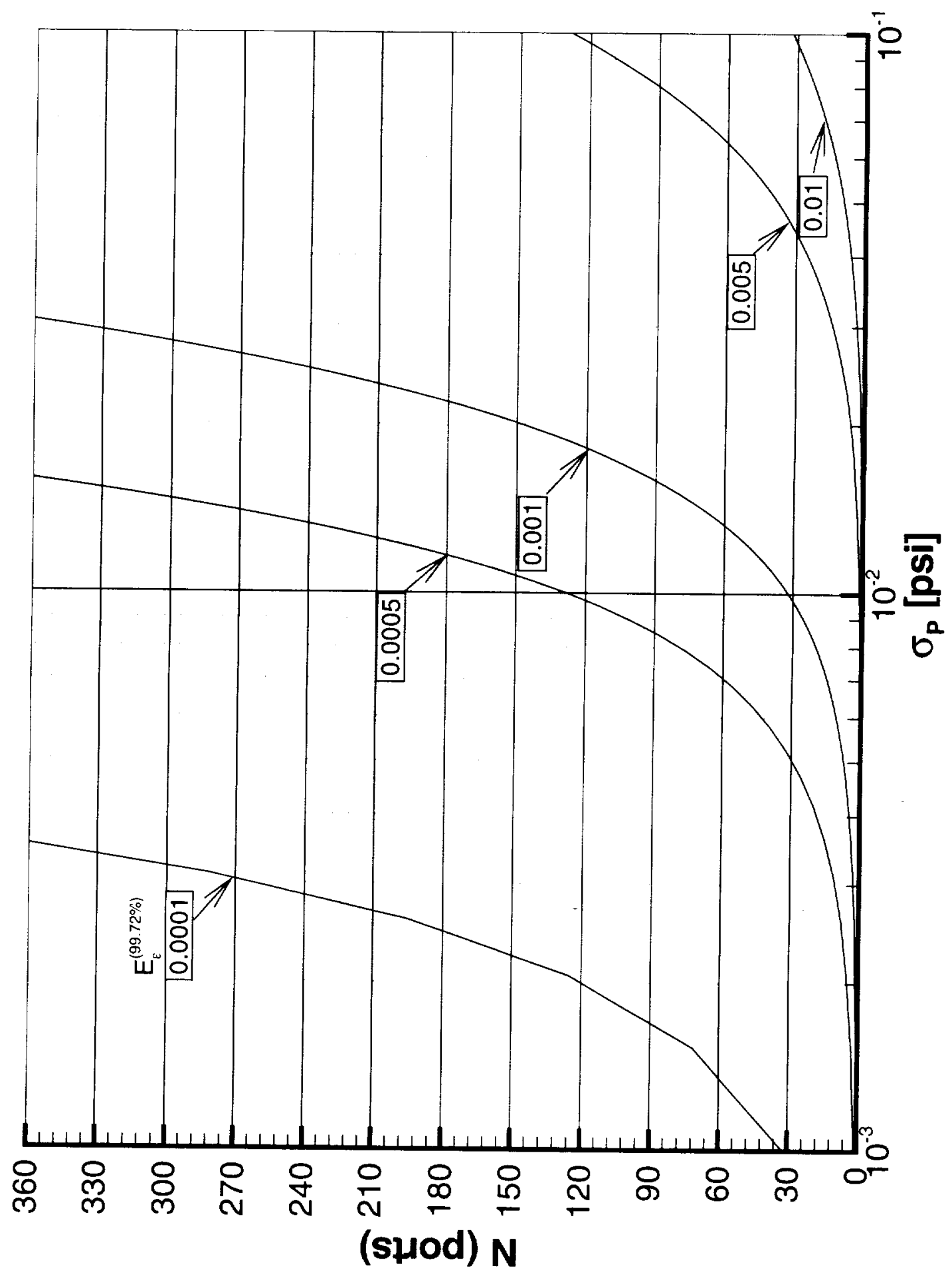

Fig. $7 \varepsilon$ Error Prediction

17 of 27 


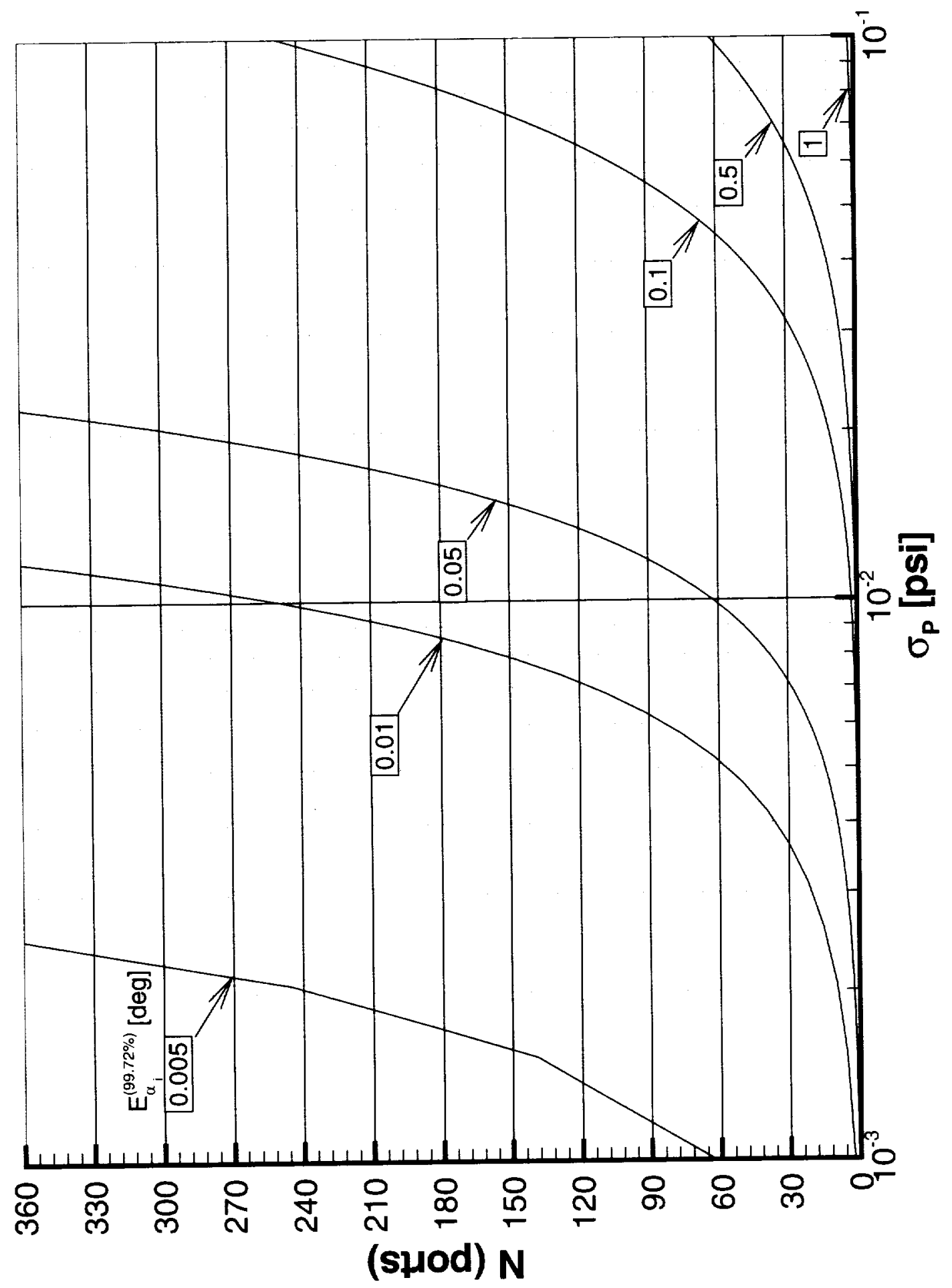

Fig. $8 \alpha_{i}$ Error Prediction

18 of $2 T$ 


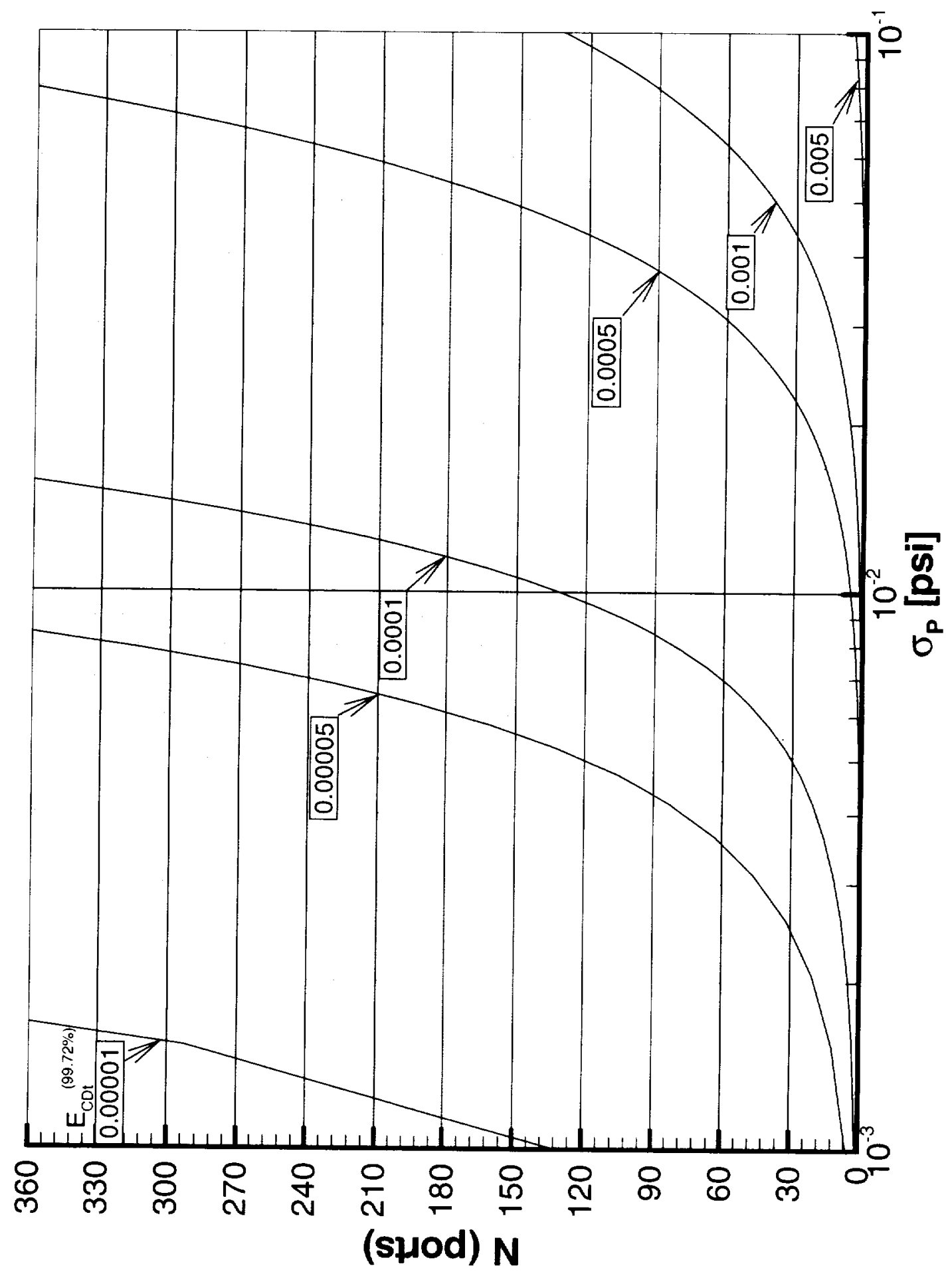

Fig. $9 \Delta C_{D_{t}}$ Error Prediction

19 of 27 


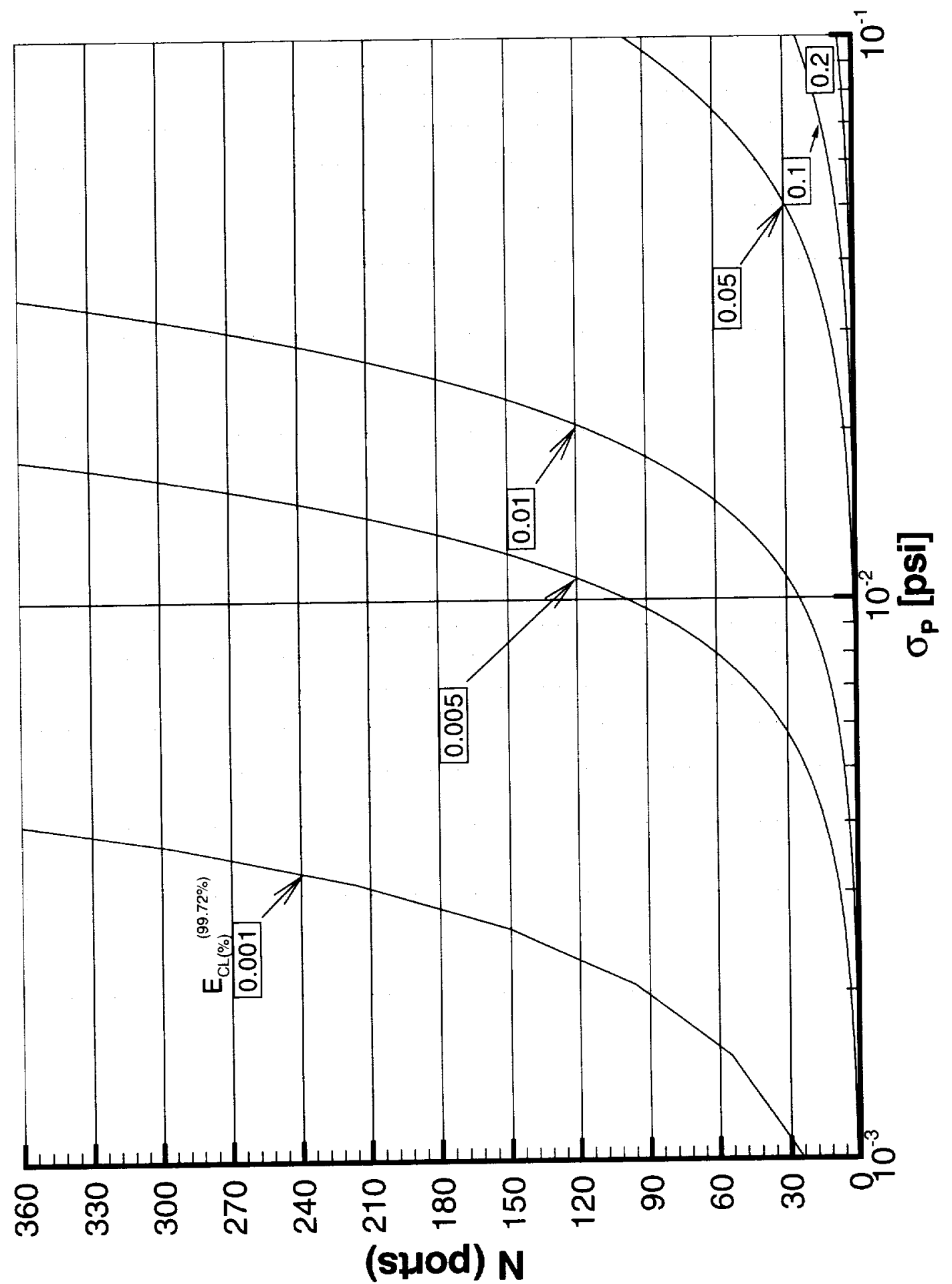

Fig. $10 \Delta C_{L(\%)}$ Error Prediction

20 of 27 


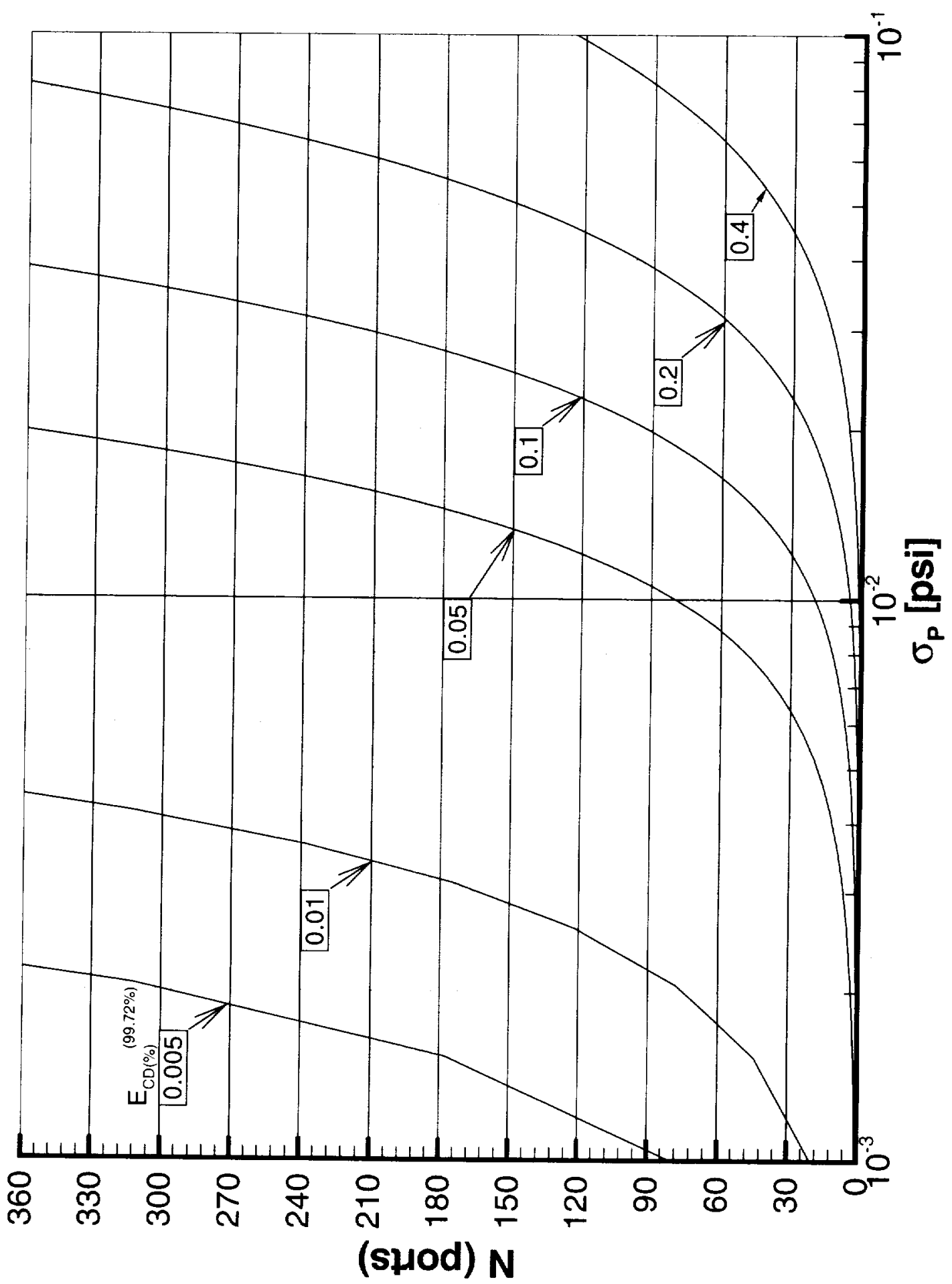

Fig. $11 \Delta C_{D_{t}(\%)}$ Error Prediction 


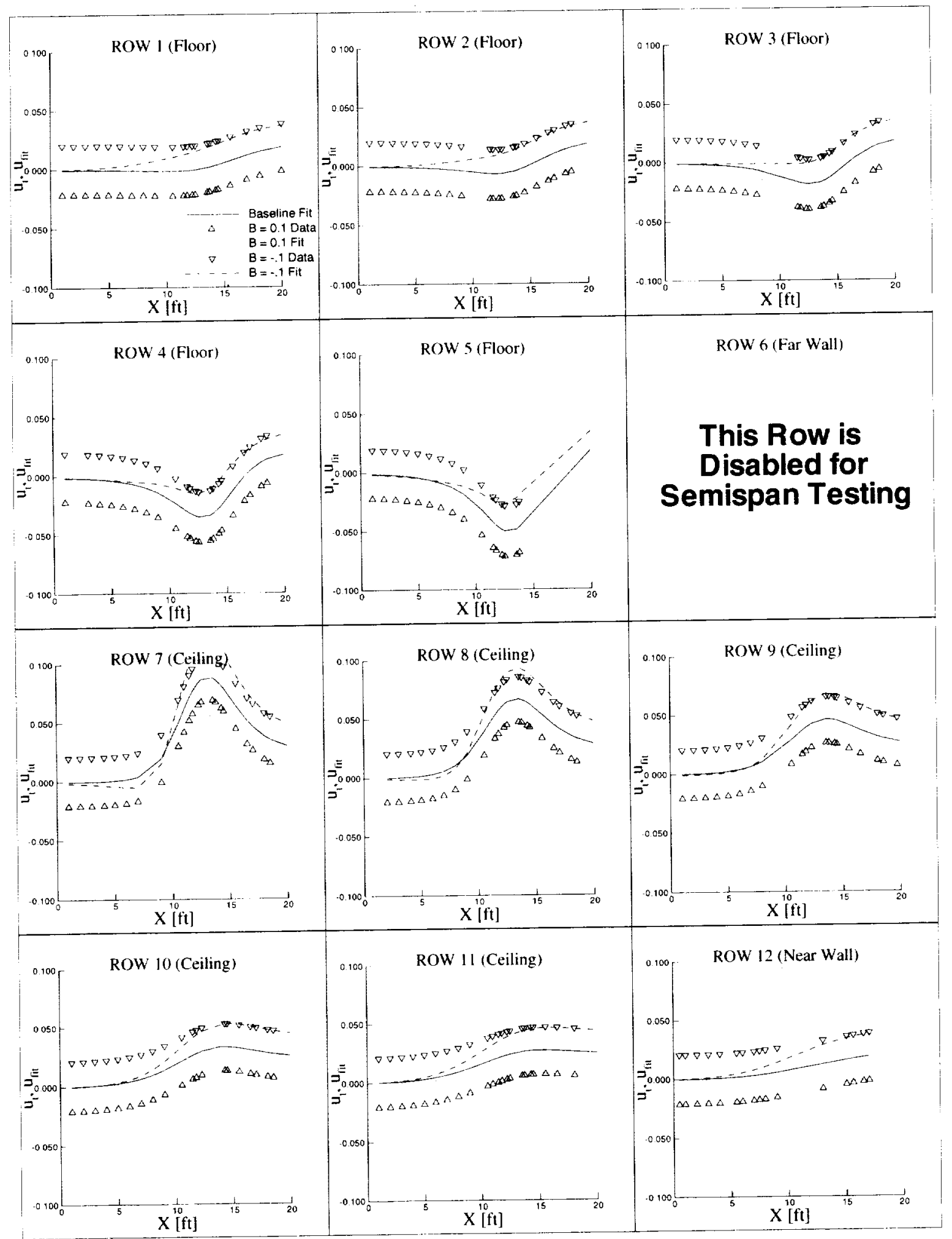

Fig. 12 Wall Signature Comparison for $B_{P}= \pm 0.1$ psi 


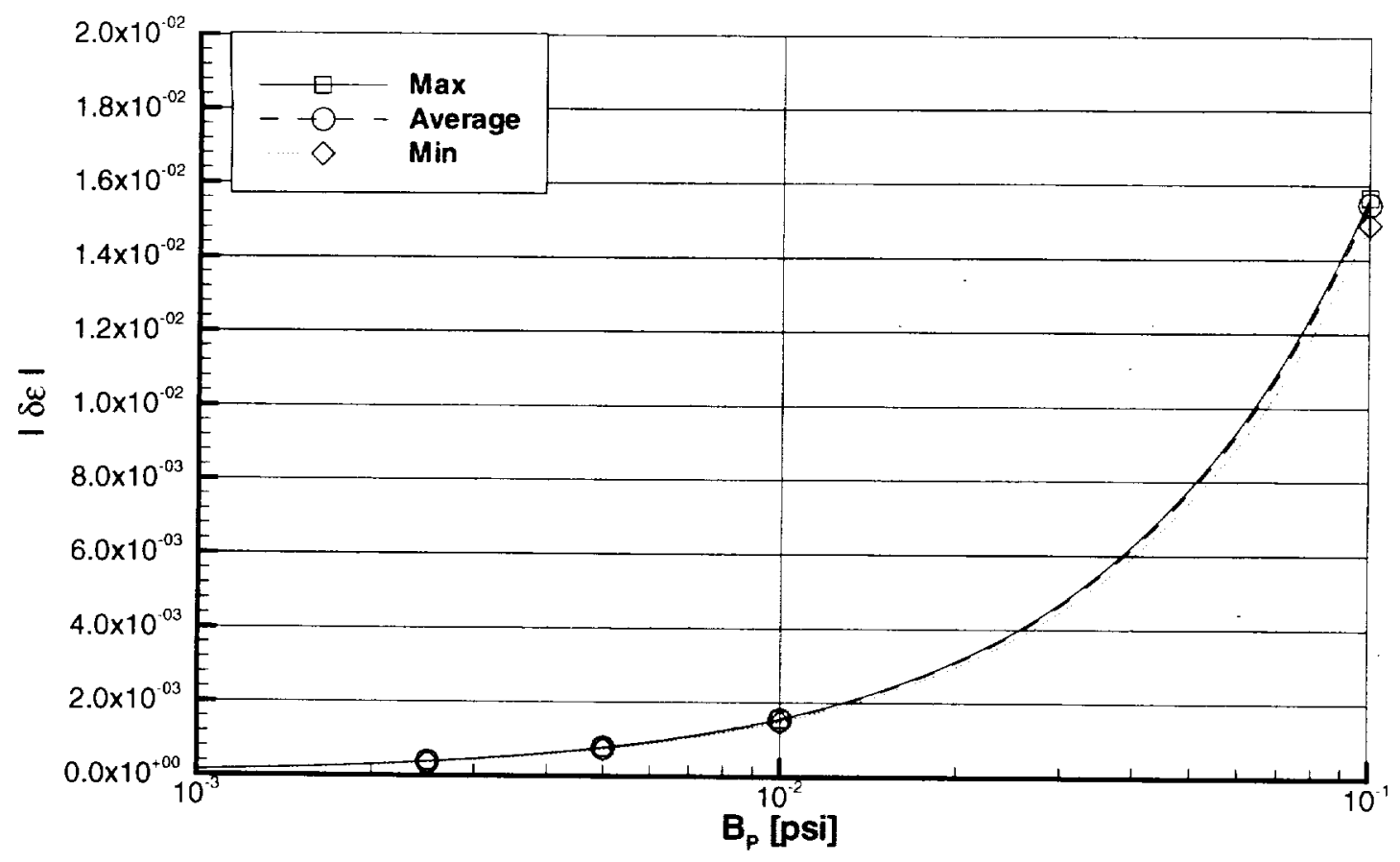

(a) Error in Blockage Factor

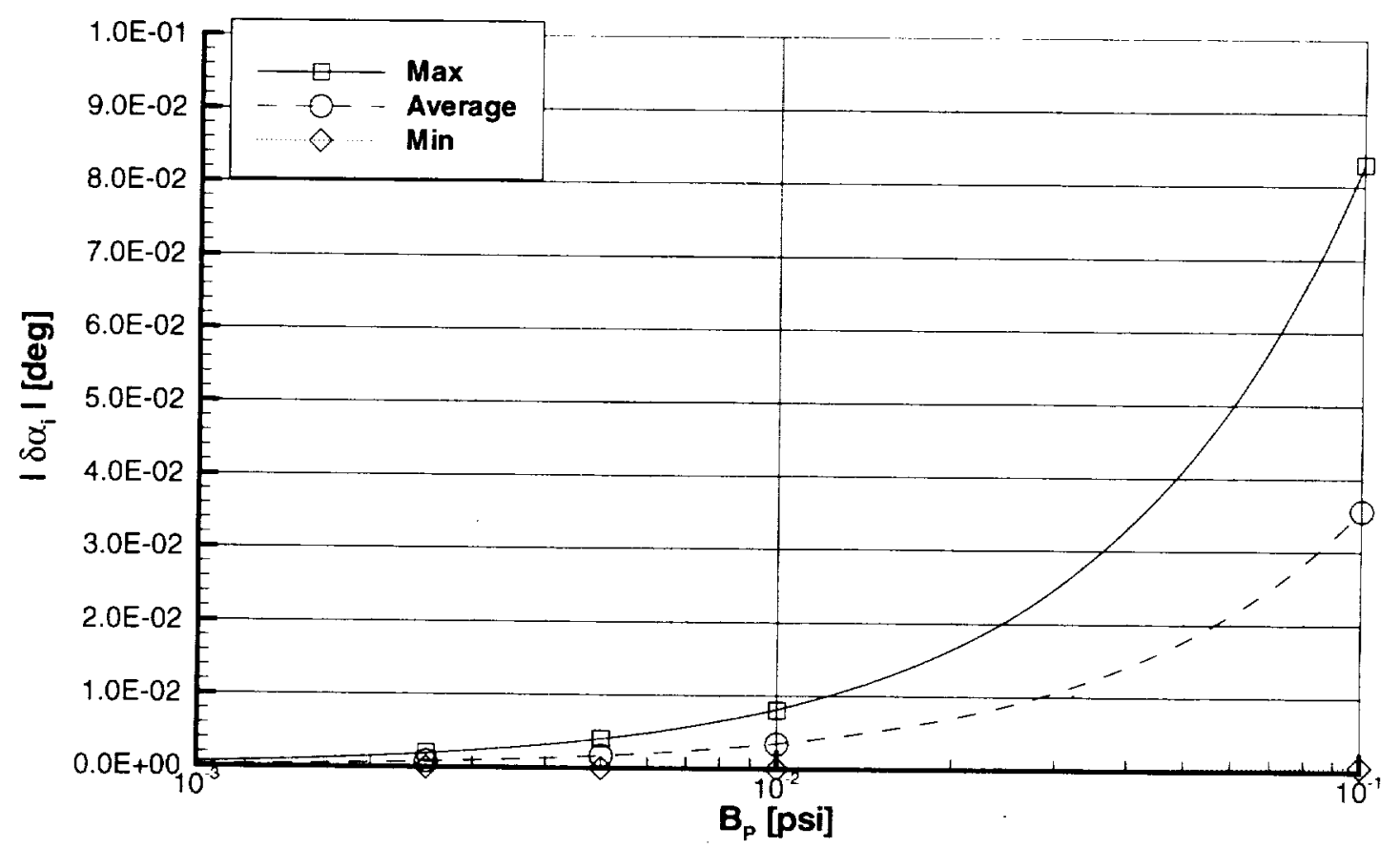

(b) Error in Upwash Correction

Fig. 13 Primary Correction Sensitivity to Variation in $B_{P}$ 


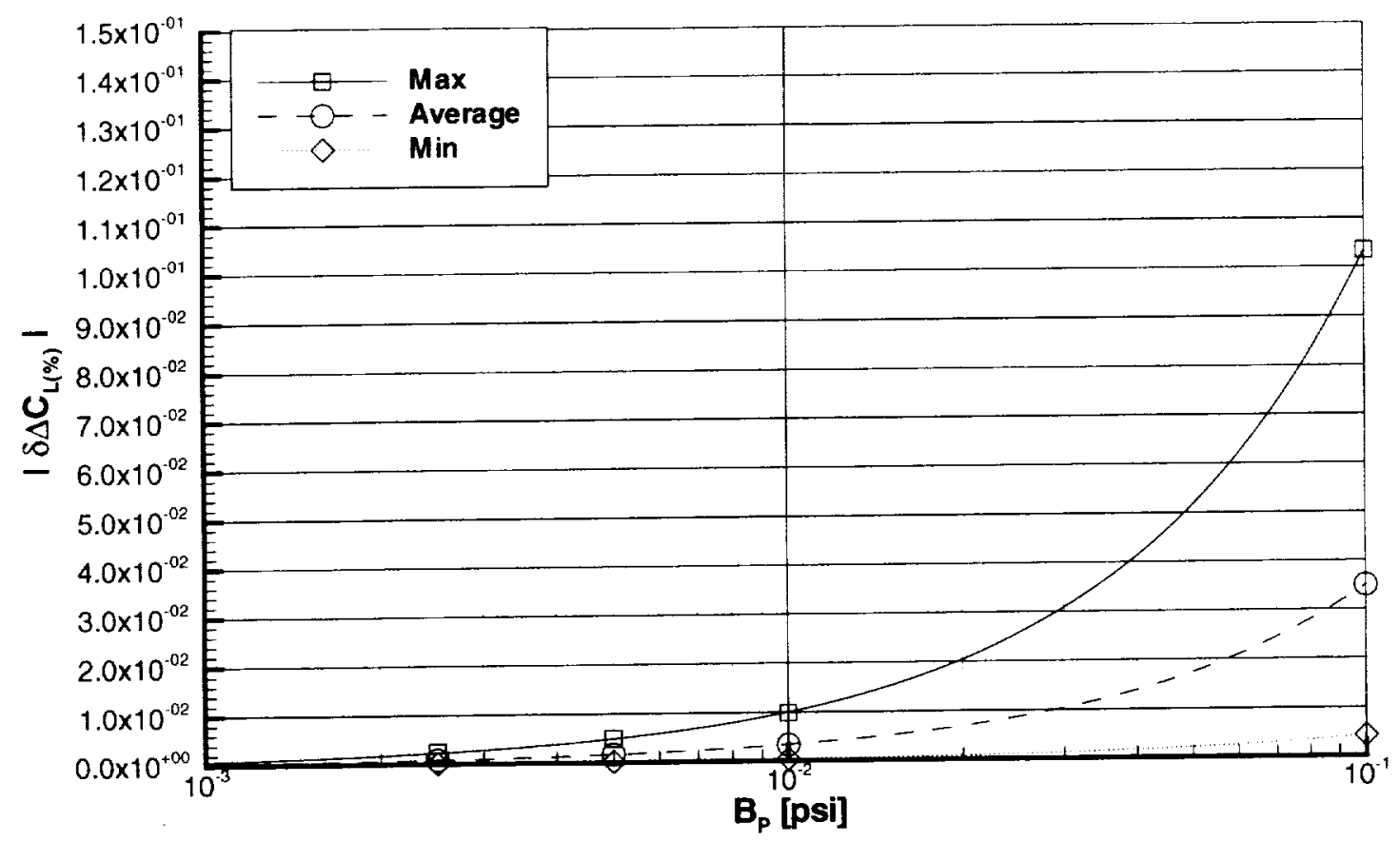

(a) Error in $\Delta C_{L}$ in Percent $C_{L \text { unc }}$

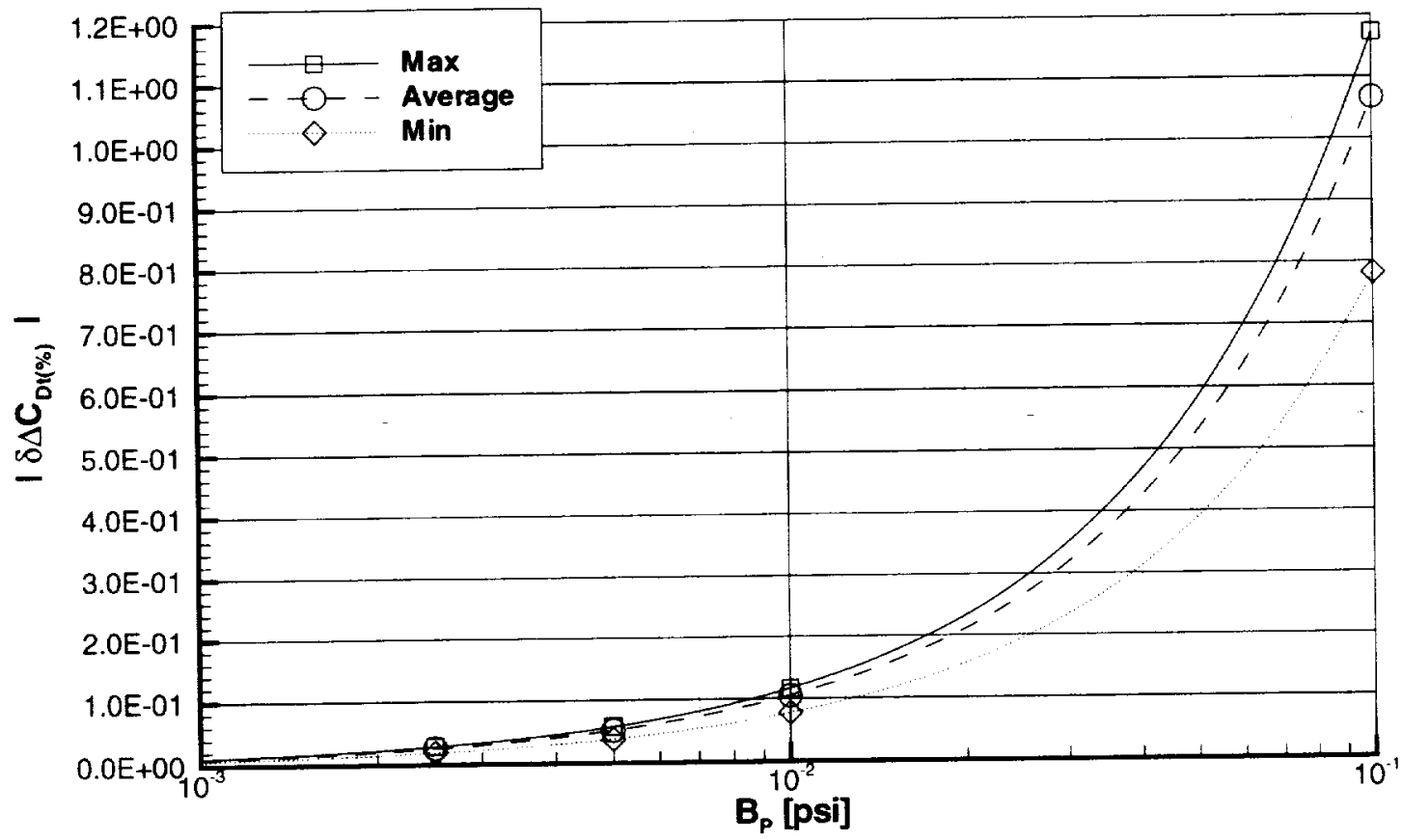

(a) Error in $\Delta C_{D t}$ in Percent $C_{D \text { unc }}$

Fig. 14 Percent Force Coefficient Correction Sensitivity to Variation in $B_{P}$ 


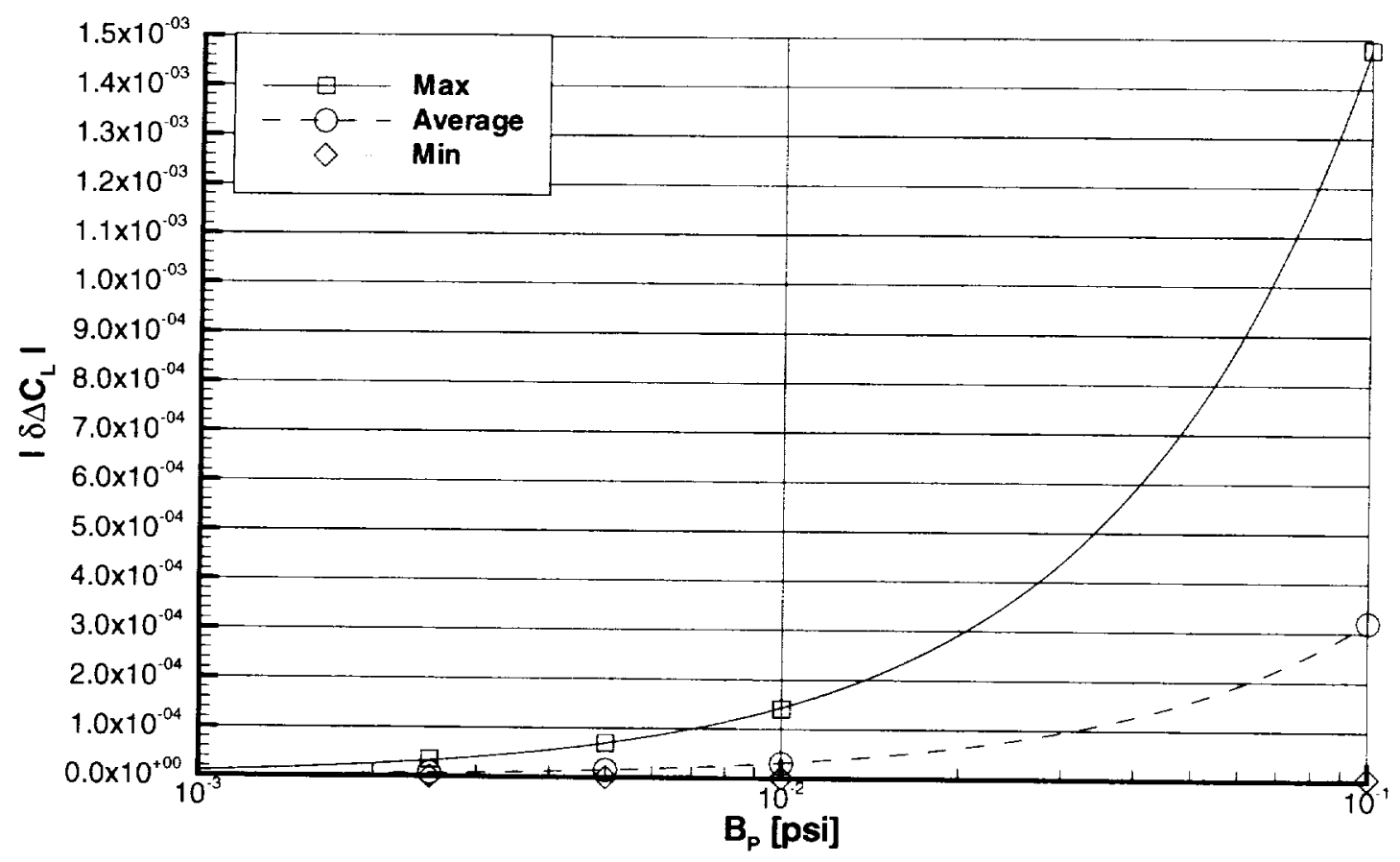

(a) Error in $\Delta C_{L}$

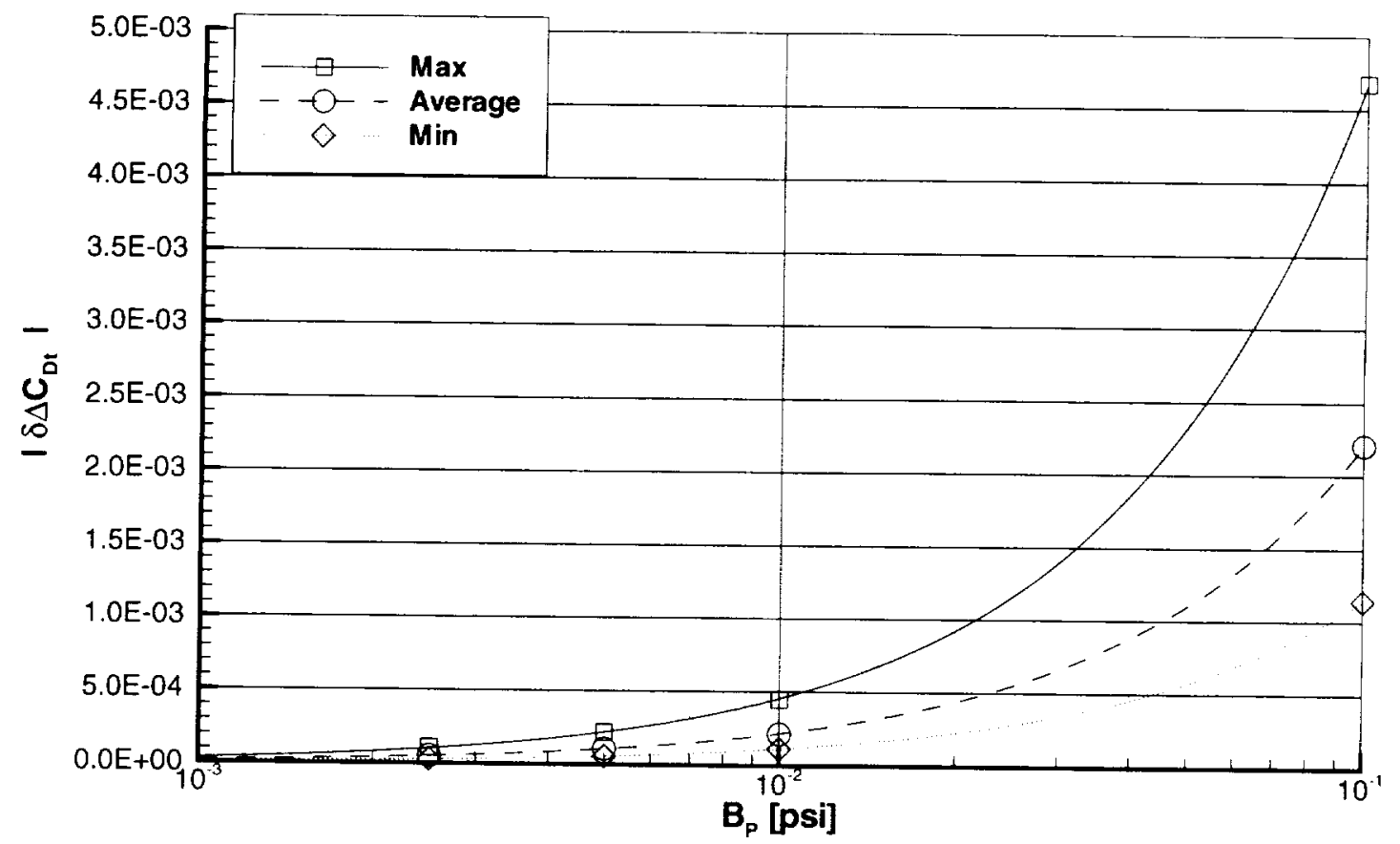

(b) Error in $\Delta \mathrm{C}_{\mathrm{Dt}}$

Fig. 15 Force Coefficient Correction Sensitivity to Variation in $B_{P}$ 


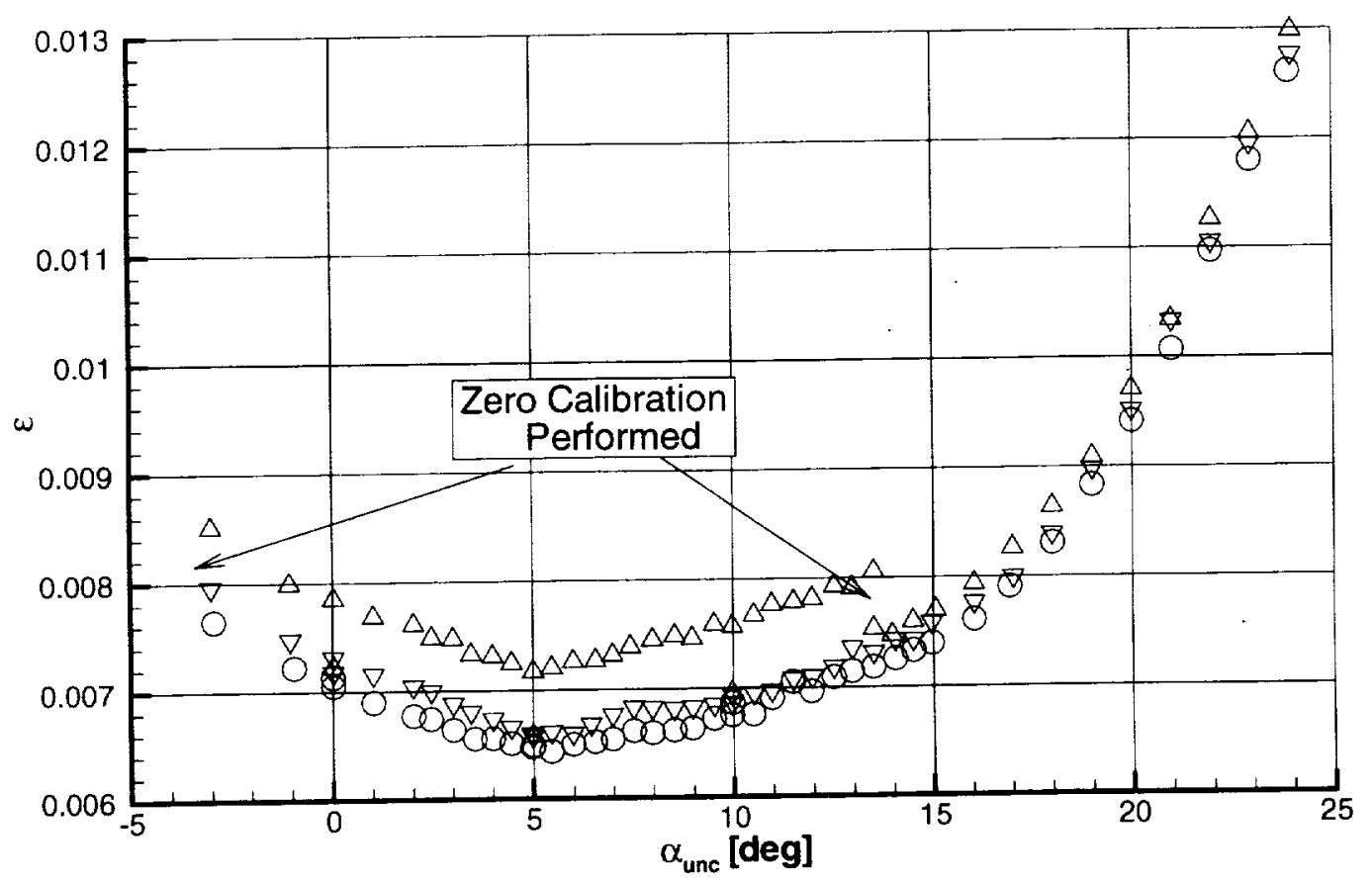

(a) Biased Case

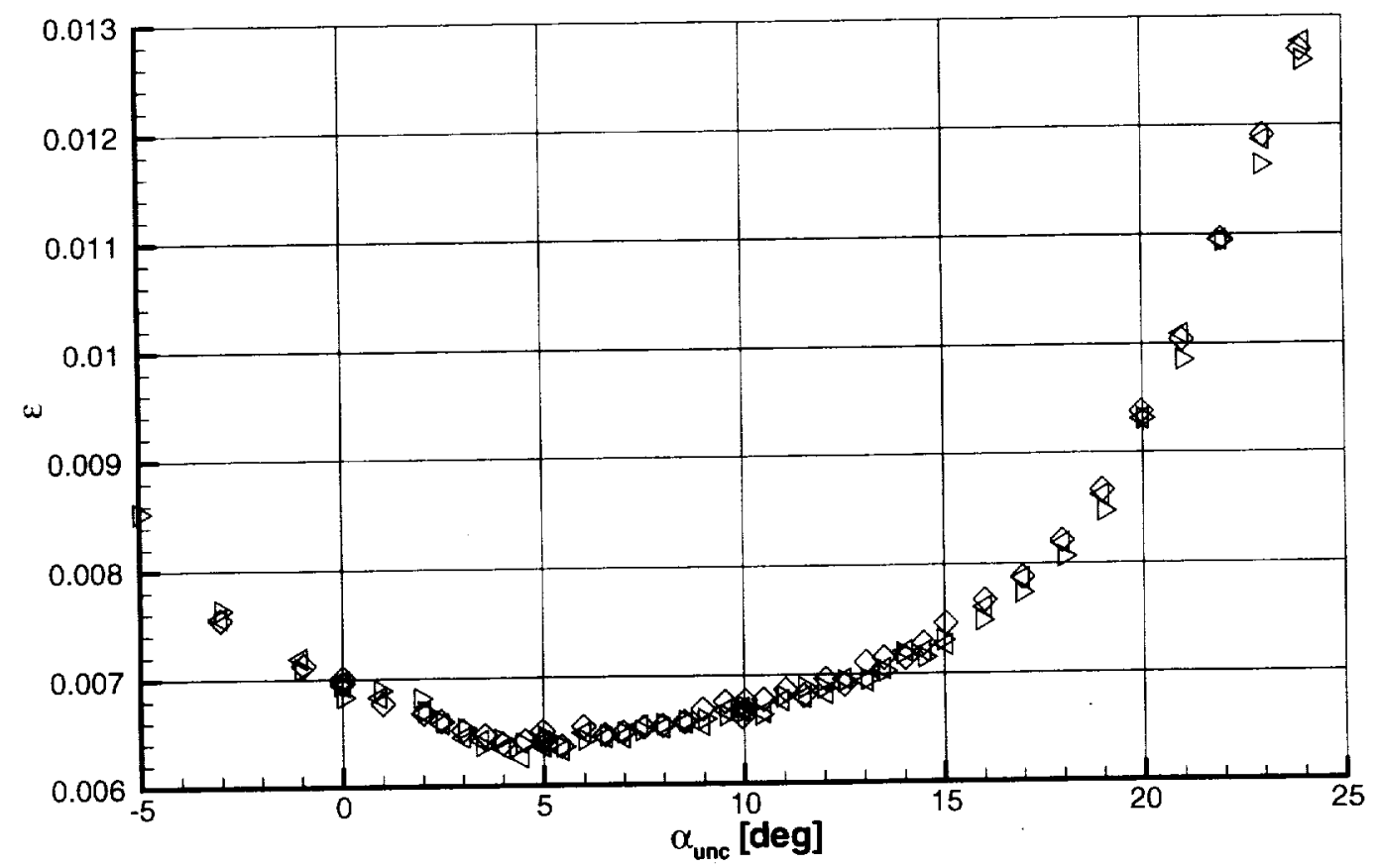

(b) Unbiased Case

Fig. 16 Biasing of Wall Pressures due to Calibration

26 of 27 


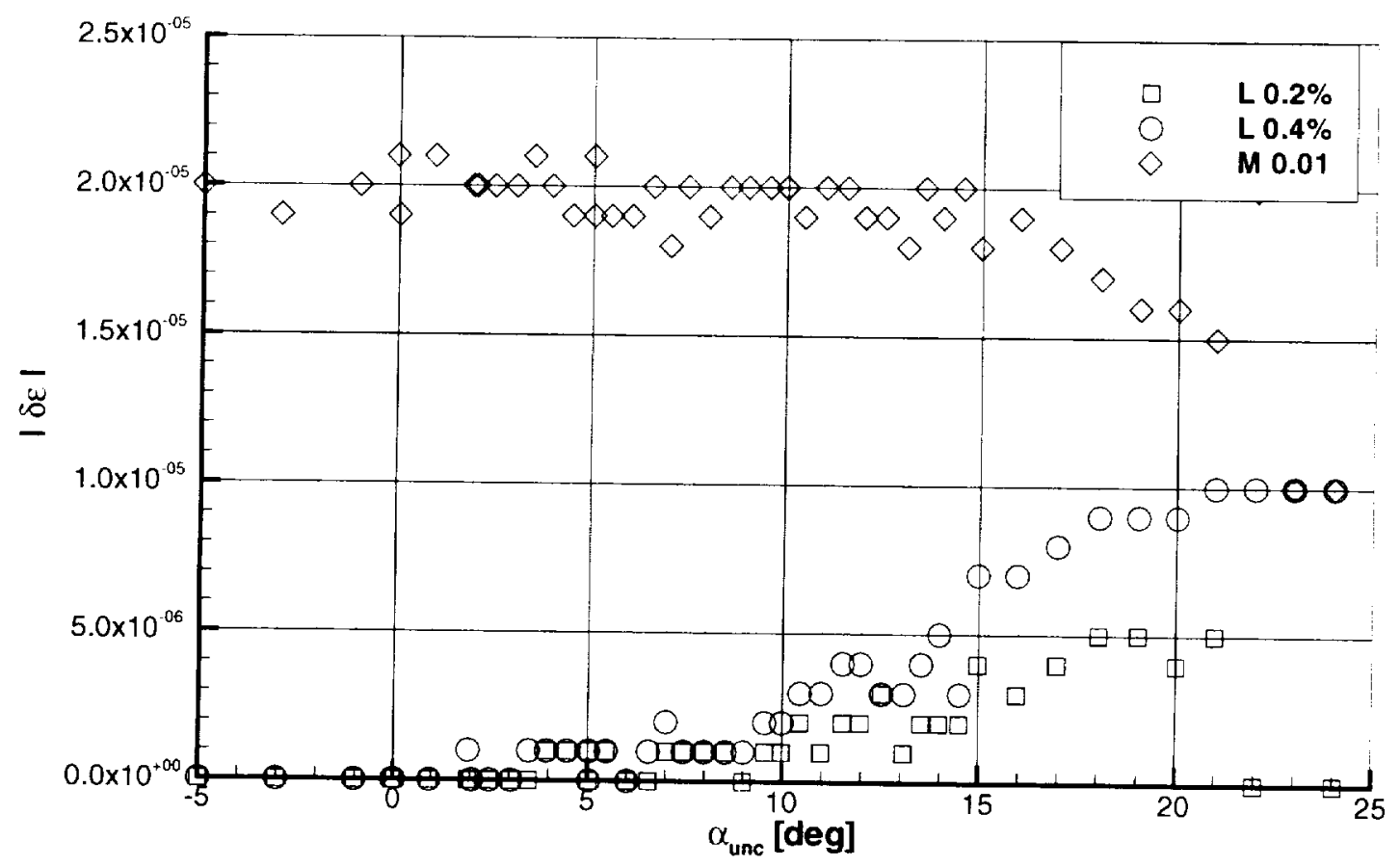

(a) Error in Blockage Factor

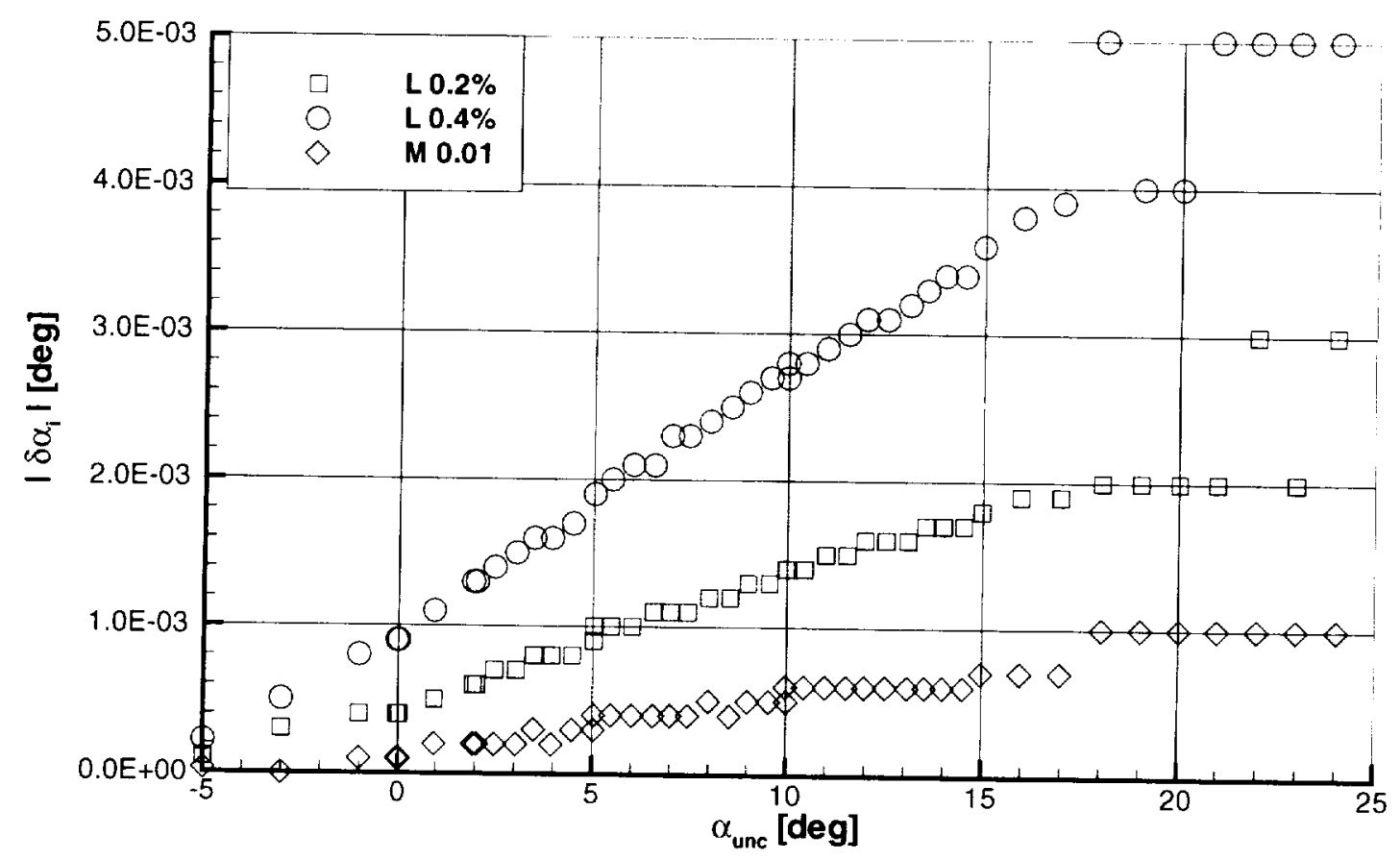

(b) Error in Upwash Correction

Fig. 17 Primary Correction Error for Other Error Sources 
-

.

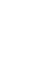

